\title{
Decorrelation and phase-shift of coda waves induced by local changes: multiple scattering approach and numerical validation
}

\author{
Thomas Planès ${ }^{\mathrm{a} *}$, Eric Larose ${ }^{\mathrm{a}}$, Ludovic Margerin ${ }^{\mathrm{b}}$, Vincent Rossetto ${ }^{\mathrm{c}}$ and \\ Christoph Sens-Schönfelder ${ }^{\mathrm{d}}$
}

\author{
${ }^{a}$ ISTerre Université de Grenoble 1 and CNRS, Grenoble, France; ${ }^{b}$ IRAP/CNRS, Université \\ de Toulouse, Toulouse, France; ${ }^{c}$ LPMMC/CNRS, Université de Grenoble 1, Grenoble, France; \\ ${ }^{d}$ GFZ German Research Centre for Geosciences, Potsdam, Germany
}

\begin{abstract}
We report on theoretical predictions of the decorrelation and phase-shift of coda waves induced by local changes in multiple scattering media. Using the multiple scattering formalism, we show that both expressions (decorrelation and phaseshift) involve a same sensitivity kernel based on the intensity transport in the medium. We compare the kernels based on the diffusion approximation with the ones based on the radiative transfer approximation, showing that the latter is more accurate at short times or for changes located close to the source or the receiver. We also perform a series of numerical simulations of wave propagation (finite differences) to validate our models in different configurations.
\end{abstract}

\section{Introduction}

Heterogeneous materials excited by waves are known to show complex long-lasting signals that may appear random. These responses have long been regarded as being devoid of any practical information about the medium of propagation, and sometimes even considered as 'noise.' Nevertheless, these acoustic, elastic, or electromagnetic wave trains are perfectly reproducible: the random nature of the Green's functions does not originate from the wave source or from the propagation equation, but from the random spatial distribution of the physical properties of the material. These long-lasting wave trains, forming the tails of the record, can be observed in many domains of wave physics: in optics (a laser pointing into a glass of milk for instance), in acoustics (ultrasound propagating through a collection of steel rods immersed in water,[1] or in concrete [2]), in seismology,[3] etc. In the latter case, the vibrations arriving late after the direct waves were called 'coda' waves,[4] a terminology now adopted in many other domains.

The scattering mean free path $\ell$ is the distance that quantifies the degree of heterogeneity of the material, and is related to the distance between two successive scattering events. The higher the degree of heterogeneity, the shorter the mean free path. This mean free path is defined as the characteristic length of exponential attenuation of the coherent wave,

*Corresponding author. Present address: Department of Civil and Environmental Engineering, Colorado School of Mines, Golden, CO, USA. Email: tplanes@mines.edu 
in absence of intrinsic absorption. The coherent wave is the wave that resists ensemble averaging (when such an averaging is practically possible). When looking at the decay of the averaged intensity (not the amplitude), the pertinent length is the transport mean free path $\ell^{\star}$, which corresponds to the distance after which the wave has lost the memory of its initial direction of propagation. In practical applications, these two quantities are to be compared to other characteristic lengths, including primarily the wavelength $\lambda$ and the intrinsic absorption length $\ell_{a}$. In the single scattering regime, where most communication, imaging and monitoring techniques operate, we have the following scaling:

$$
\lambda \ll \ell_{a}<\ell
$$

which means that the wave is attenuated before being scattered several times by the heterogeneities. When the degree of heterogeneity of the medium increases, we move to the so-called 'mesoscopic' regime where multiple scattering is observed [5-8]:

$$
\lambda \ll \ell<\ell_{a}
$$

In this article, we will focus on this regime, which concerns many practical situations in everyday life. The last regime, where the wavelength is of the order of the scattering mean free path is the strong localization regime [9-11] and will not be developed in the present article.

Among many phenomena observed in this mesoscopic regime, dynamic ones have raised a particular interest. The general idea of dynamic monitoring of multiple scattering media is to operate fixed sources and receivers while the material undergoes a slight perturbation. The time evolution of the medium is much longer than the propagation time of the wave, so that the medium is considered frozen during an acquisition, but slightly evolves between two successive acquisitions. The tremendous advantage of diffuse waves compared to direct and ballistic ones is that they are much more sensitive to weak changes in the material.

During the $80 \mathrm{~s}$ and $90 \mathrm{~s}$, several authors proposed to take advantage of multiply scattered waves to monitor dynamic media. In optics, diffusing wave spectroscopy (DWS) [12] allows to quantify the Brownian motion of particles in suspension from the observation of the dynamic decorrelation of the speckle pattern. This technique was further developed in acoustics [13] where it found many practical applications. In the case of solid media where scatterers are fixed, but where the medium undergoes global changes such as velocity variations, a different technique has been introduced in the 80s,[14] and later developed under the terminology coda wave interferometry.[15] It consists in measuring the slight velocity change of the material from the time delay or phase-shift of the waveforms in the coda. Applications in acoustics [16,17] and in seismology $[18,19]$ abound. These two techniques (D(A)WS and CWI) are designed to measure global changes in the medium. The present article addresses a different situation where the change is local.

The problem of detecting and locating an isolated change in a multiple scattering sample has received some attention in the past. In optics, the techniques proposed in the early 90s are based on the variation of intensity in a speckle pattern probed by one or more receivers.[20-22] Nieuwenhuizen and van Rossum [23] have shown that a new defect (the perturbation) added to a collection of scatterers (the background) essentially acts as a dipole source of intensity. These authors also found that this method has weak sensitivity and that a large amount of ensemble or frequency averaging (typically 100 realizations) is required to distinguish the intensity fluctuation caused by the defect from the background 
speckle pattern. This implies that techniques based on intensity variations are unlikely to be applicable to solid media.

Pacheco and Snieder [24] proposed to extend the CWI to local changes, by modeling the traveltime variations of the coda waves. This approach is well suited to detect slight velocity changes that occur in a relatively extended area. However, this approach is not adequate to describe strong and very localized changes appearing in the medium.

In this study, we aim to predict the slight changes in the diffuse waveforms (coda waves) induced by a local change in a strongly scattering medium. Depending on the strength of the change in the medium (impedance contrast), we assume that two different measurements can be relevant to quantify the change. On the one hand, we assume that a local structural change (strong impedance contrast) will mainly induce a decorrelation of the waveforms (amplitude and phase variation). On the second hand, we assume that a local slight velocity change (weak impedance contrast) will mainly induce time lapses between the waveforms (phase-shift).

Using experimental measurements of the decorrelation, Larose et al. [25] and Rossetto et al. [26] proposed a new imaging technique to locate the change in diffusive media, called Locadiff. Similarly, Froment [27] and Obermann et al. [28] used experimental phase-shift measurements to image velocity changes in active faults and volcanoes. However, the forward problem has not been fully validated yet.

The first aim of the present study is to provide a full theoretical derivation of the decorrelation and phase-shift induced by local changes (2D and 3D). We unify and extend previous studies,[24,26] using a same multiple scattering approach, and we use numerical simulations to illustrate and test our models in different configurations.

In Section 2, we describe the setup and parameters of the numerical simulations. This allows us to numerically illustrate the properties of the decorrelation induced by a local structural change.

In Section 3, to predict this decorrelation, we derive a theoretical model using the multiple scattering formalism. This model is based on a diagrammatic expansion of the correlation that takes into account the effect of a new strong scatterer. Defining the new scatterer as a slight velocity change, we then derive an expression for the time-shift induced on coda waves, confirming a result previously derived in a probabilistic sense.[24]

These two models involve a same sensitivity kernel that we study for different intensity transport approximations, in 2D and 3D (Section 4). We consider the diffusion approximation as well as the radiative transfer approximation and we discuss the differences of their corresponding kernels.

Finally, in Section 5, we test and validate the different kernels using numerical simulations of wave propagation in multiple scattering media (2D acoustic finite differences).

\section{Decorrelation of coda waves: numerical simulations}

\subsection{Simulation parameters}

To simulate the multiple scattering of waves in heterogeneous media, we perform numerical experiments of acoustic wave propagation in a medium with constant background velocity, filled with thousands of point scatterers. To solve the wave equation (here for the pressure field), we use a classic second-order 2D finite difference scheme ([2,2] FDTD).[29] In the following, spatial quantities are expressed in units of $\lambda_{0}$, the central wavelength, and 
Table 1. Simulation parameters: dimensionless values, values that correspond to ultrasound simulation and values that correspond to seismic wave simulation.

\begin{tabular}{lcccl}
\hline Notation & Dimensionless & US & Seismics & Description \\
\hline$f_{0}$ & $1 / \tau_{0}$ & $500 \mathrm{kHz}$ & $10 \mathrm{~Hz}$ & Central frequency \\
$c_{0}$ & $\lambda_{0} / \tau_{0}$ & $2500 \mathrm{~m} \cdot \mathrm{s}^{-1}$ & $3900 \mathrm{~m} \cdot \mathrm{s}^{-1}$ & Wave speed \\
$\lambda_{0}$ & $\lambda_{0}$ & $0.5 \mathrm{~cm}$ & $390 \mathrm{~m}$ & Central wavelength \\
$\Delta f$ & $30 \%$ & & & Relative Bandwidth \\
$a$ & $\lambda_{0} / 15$ & $333 \mu \mathrm{m}$ & $26 \mathrm{~m}$ & Spatial grid step \\
$\eta$ & $0.0471 \tau_{0}$ & $94.2 \mathrm{~ns}$ & $4.71 \mathrm{~ms}$ & Temporal step \\
$L$ & $200 \lambda_{0}$ & $1 \mathrm{~m}$ & $78 \mathrm{~km}$ & $\begin{array}{l}\text { Medium length } \\
\sigma_{0}=\sigma_{0}^{\star}\end{array}$ \\
$0.174 \lambda_{0}$ & $0.87 \mathrm{~mm}$ & $68 \mathrm{~m}$ & $\begin{array}{l}\text { Scattering and transport } \\
\text { cross-section of a point scatterer } \\
\ell=\ell^{\star}\end{array}$ \\
$10 \lambda_{0}$ & $5 \mathrm{~cm}$ & $3.9 \mathrm{~km}$ & Scattering and transport \\
& & & & mean free path \\
$N$ & 23,000 & & & Number of point scatterers \\
& & & & in the medium \\
\hline
\end{tabular}

temporal quantities are expressed in units of $\tau_{0}$, the central period. We consider a squareshaped lossless medium with reflective boundaries (Dirichlet boundary condition), of length $L=200$, constant velocity $c_{0}=1$, in which a point source sends a Gaussian impulse of central frequency $f_{0}=1$ and relative bandwidth $\Delta f=30 \%$ (at $-3 \mathrm{~dB}$ ). The spatial gridstep is $a=1 / 15$ and the time step of the scheme is set to $\eta=a /\left(c_{0} \sqrt{2}\right) \simeq 0.0471$ using the Courant-Friedrichs-Lewy stability condition.[30]

To simplify the theory later developed, we choose to work within the independent scattering approximation (ISA) which concerns dilute systems, i.e. where the scattering mean free path is much larger than the central wavelength $\ell \gg \lambda_{0}$. We determine the number of scatterers in the medium so that $\ell=10$. Each soft scatterer is defined by a nullpressure condition at its grid point. From incident plane wave simulations, we evaluated the scattering cross-section $\sigma=0.174$ of a point scatterer. The number $N$ of scatterers to place in the medium is then deduced from the usual relation:

$$
\ell=\frac{1}{n \sigma}
$$

where $n=N / V$ is the density of scatterers, $V=L^{2}$ being the volume of the medium. The scatterers are randomly positioned in space and uncorrelated. Given their small size compared to the wavelength, the scattering and transport cross-sections of the scatterers are identical $\left(\sigma=\sigma^{\star}\right)$. This means that the scattering is isotropic and it also implies the equality of the scattering and transport mean free paths $\left(\ell=\ell^{\star}\right)$. All the relevant simulation parameters are summarized in the Table 1 . We also added typical values of the key physical parameters for both ultrasound and seismic waves, which constitute two possible fields of application of the physics presented in this paper.

A typical realization of the disorder is shown in Figure 1(a) where the dots, the asterisk and the cross represent the point scatterers, the source and the receiver, respectively. 

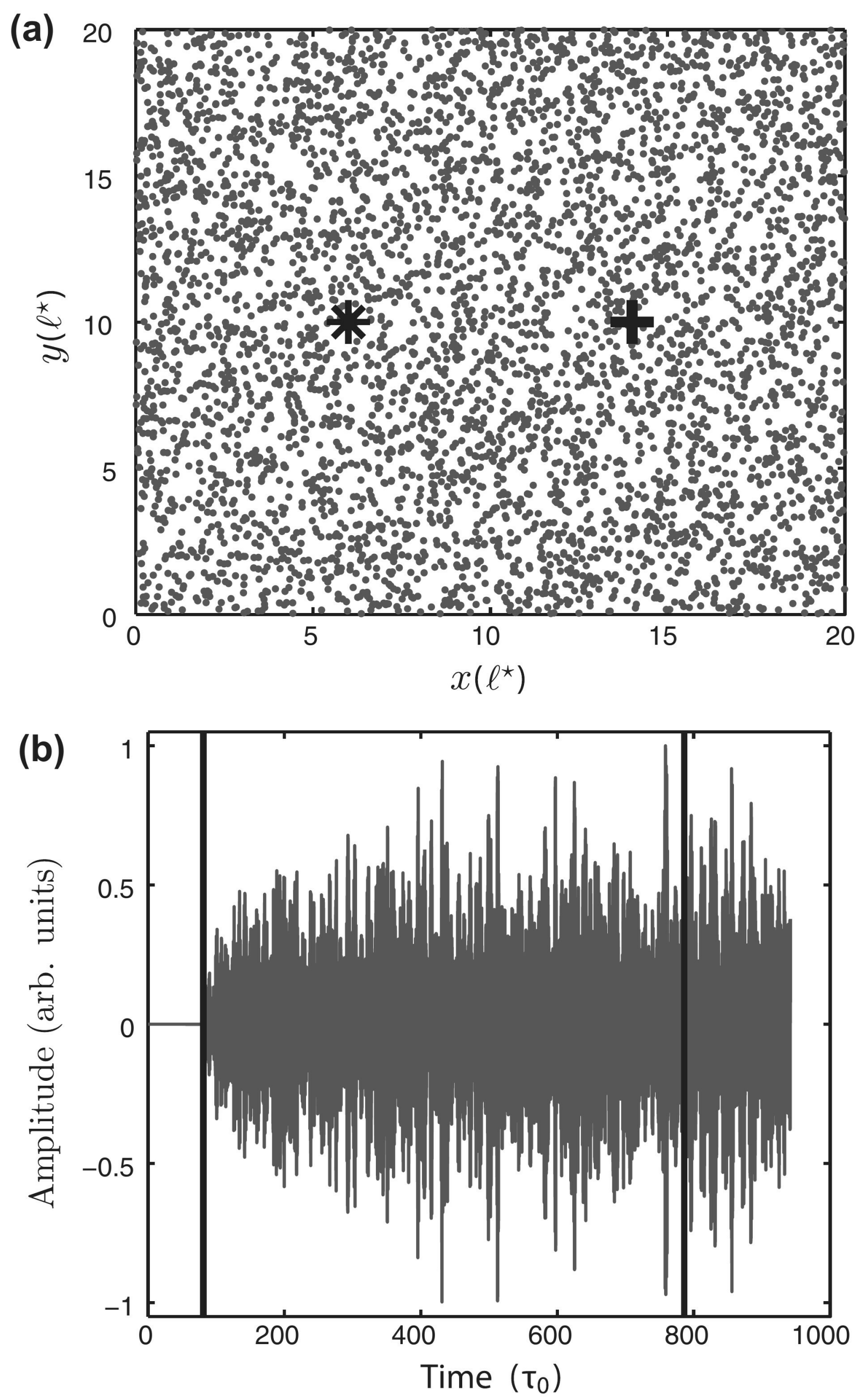

Figure 1. Typical medium (a): the dots represent the point scatterers, the asterisk the source, and the cross the receiver. The corresponding impulse response (b) shows a long tail (coda) constituted by multiply scattered waves. The two vertical lines denote the position of the zooms in Figure 2.

The corresponding impulse response is also displayed in Figure 1(b). The impulse response shows long-lasting wave trains constituted of multiply scattered waves.

\subsection{Decorrelation of the diffuse field}

To study the variations of the waveforms induced by a local change in the medium, we compare impulse responses acquired before and after the change. Early and late timelapse zooms of the impulse response associated to the initial state are plotted in Figure 2(b) (solid line). To simulate a local structural change, we introduce a new scatterer in the medium, either between the source and receiver or off the source-receiver axis (see Figure 2(a)). In both cases, the final impulse responses are different from the initial one 
(see Figure 2(b)). At early times, the waveforms are almost perfectly matching each other. However, at long times, we observe slight differences in phase and amplitude of the coda waveforms. The addition of a defect results in both distortion and travel-time perturbations of the waveforms. These waveform variations increase with time in the coda. The longer the multiply scattered waves explore the medium, the more they interact with the change and the more the waveforms differ. Another key observation is that the variations strongly depend on the position of the change relative to the position of the source and receiver. The closer the defect to the source-receiver axis, the stronger the variation of the waveforms.

To quantify the change between an initial state of impulse response $\phi_{0}(t)$ and a final state of impulse response $\phi_{1}(t)$, we define the experimental decorrelation coefficient as:

$$
D C^{\operatorname{Exp}}(t)=1-\frac{\int_{t-\frac{T}{2}}^{t+\frac{T}{2}} \phi_{0}\left(t^{\prime}\right) \phi_{1}\left(t^{\prime}\right) d t^{\prime}}{\sqrt{\int_{t-\frac{T}{2}}^{t+\frac{T}{2}} \phi_{0}\left(t^{\prime}\right)^{2} d t^{\prime} \int_{t-\frac{T}{2}}^{t+\frac{T}{2}} \phi_{1}\left(t^{\prime}\right)^{2} d t^{\prime}}}
$$

The decorrelation corresponds to one minus the normalized correlation coefficient. It is calculated in a small time window of length $T$ and can be evaluated at different times $t$ in the coda. The size of the time window is typically a few tens of central periods $\tau_{0}$.

\section{Scattering theory of local changes}

\subsection{Estimation of the decorrelation induced by a structural change}

Let us define $g_{1}(\boldsymbol{s}, \boldsymbol{r}, t)$ and $g_{2}(\boldsymbol{s}, \boldsymbol{r}, t)$, the Green's functions of the wave equation in an initial and a final state for a source at $s$ and a receiver at $r$. The change that occurs between these states is modeled by adding an extra point scatterer at $\boldsymbol{r}_{\mathbf{0}}$. The theoretical decorrelation $D C$ induced by this change is defined as:

$$
D C\left(\boldsymbol{s}, \boldsymbol{r}, \boldsymbol{r}_{\mathbf{0}}, t\right)=1-\frac{\overline{g_{2}(\boldsymbol{s}, \boldsymbol{r}, t) g_{1}^{*}(\boldsymbol{s}, \boldsymbol{r}, t)}}{\overline{g(\boldsymbol{s}, \boldsymbol{r}, t) g^{*}(\boldsymbol{s}, \boldsymbol{r}, t)}}
$$

where the numerator of the fraction is the correlation between the initial and final state at time $t$ and the denominator is the intensity (either in the initial or final state). The overline represents the ensemble average operation, i.e. an average over all the possible realizations of the disorder. The previously introduced experimental decorrelation (4) is an estimator of this theoretical quantity.

We define the correlation in the temporal $(q)$ and frequency $(Q)$ domain by:

$$
\begin{gathered}
\bar{q}(t, \boldsymbol{s}, \boldsymbol{r})=\overline{g_{2}(\boldsymbol{s}, \boldsymbol{r}, t) g_{1}^{*}(\boldsymbol{s}, \boldsymbol{r}, t)} \\
\bar{Q}(\Omega, \boldsymbol{s}, \boldsymbol{r})=\frac{1}{2 \pi} \int_{-\infty}^{+\infty} \overline{G_{2}^{+}\left(\boldsymbol{s}, \boldsymbol{r}, \omega+\frac{\Omega}{2}\right) G_{1}^{-}\left(\boldsymbol{s}, \boldsymbol{r}, \omega-\frac{\Omega}{2}\right)} d \omega
\end{gathered}
$$

where $G_{2}^{+}$and $G_{1}^{-}$denote the retarded Green's function of the final state and the advanced Green's function of the initial state, respectively, in the frequency domain. See (A1) for the convention employed to define the temporal Fourier transform. In the following, we omit the frequency dependences of $G$ to alleviate the notations. To evaluate the product $\overline{G_{2}^{+}(s, r) G_{1}^{-}(s, r)}$, it is necessary to replace the Green's functions by their perturbative expansions (see Appendix 1, Figure A1). We can formally write: 

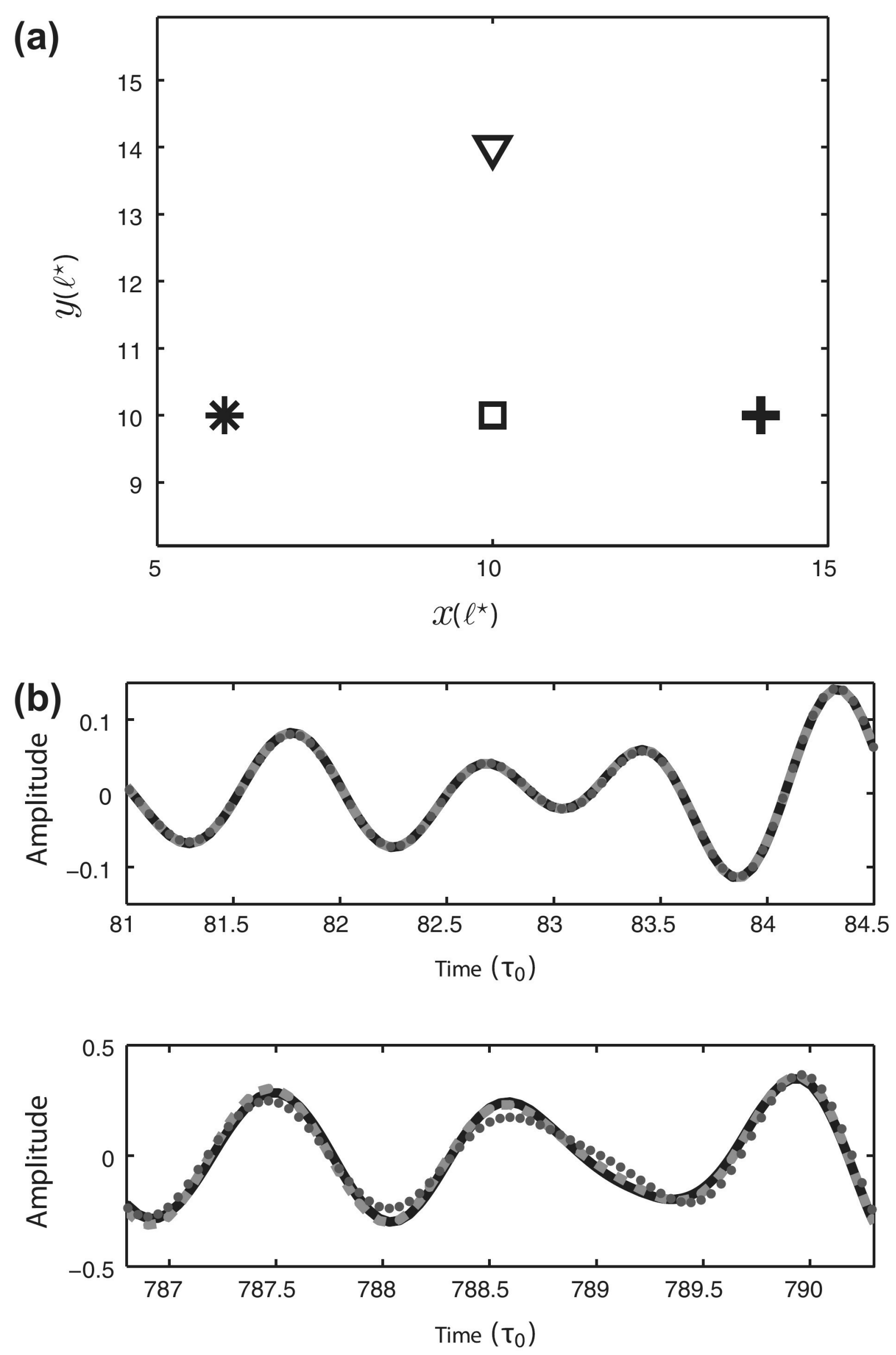

Figure 2. (a) Position 1 (square) and position 2 (triangle) where a new scatterer is added among the reference scattering medium. (b) Initial impulse response (solid line), final impulse response (dotted line) with a new scatterer in position 1, final impulse response (dashed line) with scatterer in position 2. The early (top) and late (bottom) time zooms are centered at the times indicated in Figure 1(b).

$$
\begin{aligned}
\overline{G_{2}^{+}(\boldsymbol{s}, \boldsymbol{r}) G_{1}^{-}(\boldsymbol{s}, \boldsymbol{r})}= & \bar{G}^{+}(\boldsymbol{s}, \boldsymbol{r}) \bar{G}^{-}(\boldsymbol{s}, \boldsymbol{r}) \\
& +\iint \bar{G}^{+}\left(\boldsymbol{s}, \boldsymbol{r}_{\mathbf{1}}\right) \bar{G}^{-}\left(\boldsymbol{s}, \boldsymbol{r}_{1}\right) L_{\Omega}^{\prime}\left(\boldsymbol{r}_{\mathbf{1}}, \boldsymbol{r}_{2}\right) \bar{G}^{+}\left(\boldsymbol{r}_{\mathbf{2}}, \boldsymbol{r}\right) \bar{G}^{-}\left(\boldsymbol{r}_{\mathbf{2}}, \boldsymbol{r}\right) d \boldsymbol{r}_{1} d \boldsymbol{r}_{\mathbf{2}}
\end{aligned}
$$

where we introduce a modified Ladder operator $L_{\Omega}^{\prime}\left(\boldsymbol{r}_{1}, \boldsymbol{r}_{2}\right)$ that takes into account the presence of the extra scatterer in the final state. Following previous work from Nieuwenhuizen and van Rossum [23] and assuming that the defect is weak enough, we express this modified operator using first-order perturbation theory as shown in Figure 3.

In general, the modified Ladder $L_{\Omega}^{\prime}$ is not accessible analytically. To simplify calculations, we consider a defect that is small enough to be considered point-like compare to $\lambda_{0}$. 


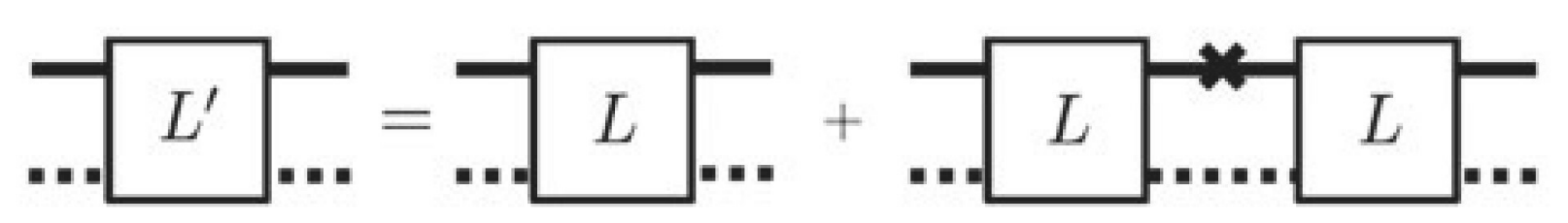

Figure 3. Diagrammatic representation of the modified Ladder operator $L^{\prime}$. The solid (resp. dashed) horizontal lines represent the average retarded (resp. advanced) Green's function $\overline{G^{+}}$(resp. $\overline{G^{-}}$) and the cross represents the $\boldsymbol{T}$-matrix of the new scatterer appearing in the medium. Here we assume that the new scatterer is far enough from the source and the receiver (a few $\ell$ ).

The $\boldsymbol{T}$-matrix [31] of the defect is thus expressed as:

$$
\mathfrak{t}_{\mathrm{o}}\left(\omega, \boldsymbol{r}_{\mathbf{1}}, \boldsymbol{r}_{\mathbf{2}}\right)=\mathfrak{t}_{\mathrm{o}}(\omega) \delta\left(\boldsymbol{r}_{\mathbf{1}}-\boldsymbol{r}_{\mathbf{0}}\right) \delta\left(\boldsymbol{r}_{\mathbf{2}}-\boldsymbol{r}_{\mathbf{0}}\right)
$$

Similarly to the frequency domain Green's function, the $\boldsymbol{T}$-matrix obeys the hermitian symmetry property:

$$
\mathfrak{t}_{\mathfrak{o}}(-\omega)=\mathfrak{t}_{\mathfrak{o}}^{*}(\omega)
$$

and the optical theorem [31] gives:

$$
\sigma=\frac{-\mathfrak{s t}_{\mathfrak{o}}(\omega)}{k}
$$

where $\mathfrak{\Im}$ denotes the imaginary part, $\sigma$ is the total cross-section of the new defect and $k$ the wavenumber. This relation expresses the conservation of energy in the scattering process. In the case of a point defect, the modified Ladder operator reads:

$$
\begin{aligned}
L_{\Omega}^{\prime}\left(\boldsymbol{r}, \boldsymbol{r}^{\prime}\right)= & L_{\Omega}\left(\boldsymbol{r}, \boldsymbol{r}^{\prime}\right) \\
& +\iint L_{\Omega}\left(\boldsymbol{r}, \boldsymbol{r}_{\mathbf{1}}\right) \bar{G}^{+}\left(\boldsymbol{r}_{\mathbf{1}}, \boldsymbol{r}_{\mathbf{0}}\right) \mathfrak{t}_{\mathrm{o}}(\omega) \bar{G}^{+}\left(\boldsymbol{r}_{\mathbf{0}}, \boldsymbol{r}_{\mathbf{2}}\right) \bar{G}^{-}\left(\boldsymbol{r}_{\mathbf{1}}, \boldsymbol{r}_{\mathbf{2}}\right) L_{\Omega}\left(\boldsymbol{r}_{\mathbf{2}}, \boldsymbol{r}^{\prime}\right) d \boldsymbol{r}_{\mathbf{1}} d \boldsymbol{r}_{\mathbf{2}}
\end{aligned}
$$

which is the equivalent in the spatial domain of the diagrammatic representation in Figure 3 , for a point scatterer. At large times compared to the mean free time (diffusive regime), the spatial variations of the intensity (or Ladder $L_{\Omega}$ ) are weak at the scale of the mean free path $\ell$, contrary to the fast decay of the coherent waves (or averaged Green's functions $\overline{G^{+}}$, $\overline{G^{-}}$). Thus, in the diffusive regime, we use the slowly varying envelope approximation to extract the Ladder $L$ from the integral:

$$
\begin{aligned}
L_{\Omega}^{\prime}\left(\boldsymbol{r}, \boldsymbol{r}^{\prime}\right) \simeq & L_{\Omega}\left(\boldsymbol{r}, \boldsymbol{r}^{\prime}\right) \\
& +L_{\Omega}\left(\boldsymbol{r}, \boldsymbol{r}_{\mathbf{0}}\right) \mathfrak{t}_{\mathcal{o}}(\omega) L_{\Omega}\left(\boldsymbol{r}_{\mathbf{0}}, \boldsymbol{r}^{\prime}\right) \iint \bar{G}^{+}\left(\boldsymbol{r}_{\mathbf{1}}, \boldsymbol{r}_{\mathbf{0}}\right) \bar{G}^{+}\left(\boldsymbol{r}_{\mathbf{0}}, \boldsymbol{r}_{\mathbf{2}}\right) \bar{G}^{-}\left(\boldsymbol{r}_{\mathbf{1}}, \boldsymbol{r}_{\mathbf{2}}\right) d \boldsymbol{r}_{\mathbf{1}} d \boldsymbol{r}_{\mathbf{2}} \\
\simeq & L_{\Omega}\left(\boldsymbol{r}, \boldsymbol{r}^{\prime}\right)+L_{\Omega}\left(\boldsymbol{r}, \boldsymbol{r}_{\mathbf{0}}\right) \mathfrak{t}_{\mathrm{o}}(\omega) L_{\Omega}\left(\boldsymbol{r}_{\mathbf{0}}, \boldsymbol{r}^{\prime}\right) I_{21}(\omega)
\end{aligned}
$$

where the remaining integral is the loop integral $I_{21}(\omega)$ that depends on the space dimension (see Appendix 2). By inserting $L_{\Omega}^{\prime}$ in the correlation term (8) and using once again the slowly varying envelope approximation, we obtain:

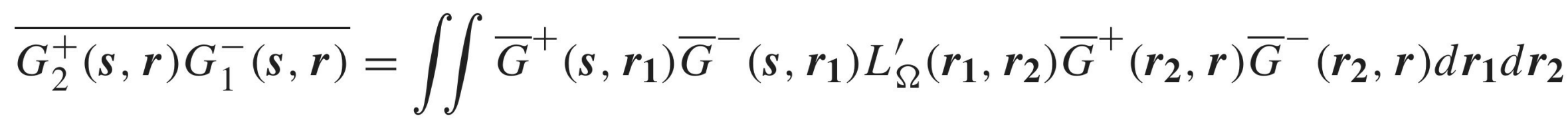

$$
\begin{aligned}
& \simeq \int \bar{G}^{+}{ }_{\left(\boldsymbol{s}, \boldsymbol{r}_{\mathbf{1}}\right) \bar{G}^{-}}\left(\boldsymbol{s}, \boldsymbol{r}_{\mathbf{1}}\right) d \boldsymbol{r}_{\mathbf{1}} L_{\Omega}^{\prime}(\boldsymbol{s}, \boldsymbol{r}) \int \bar{G}^{+}\left(\boldsymbol{r}_{\mathbf{2}}, \boldsymbol{r}\right) \bar{G}^{-}\left(\boldsymbol{r}_{\mathbf{2}}, \boldsymbol{r}\right) d \boldsymbol{r}_{\mathbf{2}} \\
& \simeq I_{11}^{2} L_{\Omega}(\boldsymbol{s}, \boldsymbol{r})+I_{11}^{2} I_{21}(\omega) \mathfrak{t}_{\mathrm{o}}(\omega) L_{\Omega}\left(\boldsymbol{s}, \boldsymbol{r}_{\mathbf{0}}\right) L_{\Omega}\left(\boldsymbol{r}_{\mathbf{0}}, \boldsymbol{r}\right)
\end{aligned}
$$


where we identify the loop integral $I_{11}$ (Appendix 2). To be consistent with the diffusive regime approximation, we also neglected the contribution of the coherent intensity from $s$ to $r$.

Using the above expression and considering signals that are filtered in a narrow frequency band $\Delta \omega$ around $\omega_{0}$, the integral (7) can be approximated as:

$$
\begin{aligned}
\bar{Q}(\Omega, \boldsymbol{s}, \boldsymbol{r}) \simeq & \frac{\Delta \omega}{\pi} I_{11}^{2} L_{\Omega}(\boldsymbol{s}, \boldsymbol{r}) \\
& +\frac{\Delta \omega}{2 \pi} I_{11}^{2}\left[I_{21}\left(-\omega_{0}\right) \mathfrak{t}_{\mathcal{o}}\left(-\omega_{\mathcal{O}}\right)+I_{21}\left(\omega_{0}\right) \mathfrak{t}_{\mathcal{o}}\left(\omega_{\mathcal{O}}\right)\right] L_{\Omega}\left(\boldsymbol{s}, \boldsymbol{r}_{\mathbf{0}}\right) L_{\Omega}\left(\boldsymbol{r}_{\mathbf{0}}, \boldsymbol{r}\right) \\
\simeq & \frac{\Delta \omega I_{11}^{2}}{\pi}\left[L_{\Omega}(\boldsymbol{s}, \boldsymbol{r})+I_{21}\left(\omega_{0}\right) i \Im \mathfrak{t}_{\mathcal{o}}\left(\omega_{\mathcal{o}}\right) L_{\Omega}\left(\boldsymbol{s}, \boldsymbol{r}_{\mathbf{0}}\right) L_{\Omega}\left(\boldsymbol{r}_{\mathbf{0}}, \boldsymbol{r}\right)\right]
\end{aligned}
$$

using hermitian symmetry of $I_{21}(\omega)(\mathrm{B} 13, \mathrm{~B} 18)$ and $\mathfrak{t}_{\mathfrak{o}}(\omega)(10)$.

To finish the calculation, we express the decorrelation (5) using the inverse Fourier transform of the correlation term (15) and the inverse Fourier transform of the intensity term (A9), over the external frequency $\Omega$ :

$$
\begin{aligned}
D C\left(\boldsymbol{s}, \boldsymbol{r}, \boldsymbol{r}_{\mathbf{0}}, t\right) & =1-\frac{T F_{\Omega}^{-1}\left[L_{\Omega}(\boldsymbol{s}, \boldsymbol{r})+i \Im \mathfrak{t}_{\mathcal{o}}\left(\omega_{\mathcal{o}}\right) I_{21}\left(\omega_{0}\right) L_{\Omega}\left(\boldsymbol{s}, \boldsymbol{r}_{\mathbf{0}}\right) L_{\Omega}\left(\boldsymbol{r}_{\mathbf{0}}, \boldsymbol{r}\right)\right]}{T F_{\Omega}^{-1}\left[L_{\Omega}(\boldsymbol{s}, \boldsymbol{r})\right]} \\
& =-i \Im \mathfrak{t}_{\mathcal{o}}\left(\omega_{\mathcal{o}}\right) I_{21}\left(\omega_{0}\right) \frac{T F_{\Omega}^{-1}\left[L_{\Omega}\left(\boldsymbol{s}, \boldsymbol{r}_{\mathbf{0}}\right) L_{\Omega}\left(\boldsymbol{r}_{\mathbf{0}}, \boldsymbol{r}\right)\right]}{T F_{\Omega}^{-1}\left[L_{\Omega}(\boldsymbol{s}, \boldsymbol{r})\right]}
\end{aligned}
$$

Using the optical theorem (11) and the relation between the Ladder $L_{\Omega}$ and the normalized intensity $I_{\Omega}$ (A9), we obtain:

$$
D C\left(\boldsymbol{s}, \boldsymbol{r}, \boldsymbol{r}_{\mathbf{0}}, t\right)=\frac{i I_{21} \sigma k_{0} c}{I_{11} \ell} \frac{T F_{\Omega}^{-1}\left[I_{\Omega}\left(\boldsymbol{s}, \boldsymbol{r}_{\mathbf{0}}\right) I_{\Omega}\left(\boldsymbol{r}_{\mathbf{0}}, \boldsymbol{r}\right)\right]}{T F_{\Omega}^{-1}\left[I_{\Omega}(\boldsymbol{s}, \boldsymbol{r})\right]}
$$

$k_{0}$ being the central wavenumber and $\sigma$ the total cross-section of the new defect. In $2 \mathrm{D}$ as well as in 3D, the ratio of the loop integrals (Appendix 2) $I_{11}$ and $I_{21}$ is given by:

$$
\frac{I_{21}}{I_{11}}=-\frac{i \ell}{2 k_{0}}
$$

The prefactor in (17) thus simplifies to $c \sigma / 2$. Applying the inverse Fourier transform to the numerator and denominator in (17) yields:

$$
D C\left(\boldsymbol{s}, \boldsymbol{r}, \boldsymbol{r}_{\mathbf{0}}, t\right)=\frac{c \sigma}{2} \frac{\int_{0}^{t} I\left(\boldsymbol{s}, \boldsymbol{r}_{\mathbf{0}}, u\right) I\left(\boldsymbol{r}_{\mathbf{0}}, \boldsymbol{r}, t-u\right) d u}{I(\boldsymbol{s}, \boldsymbol{r}, t)}
$$

where $I(t)$ is the normalized diffusive intensity in the time domain (A10). The convolution term in time arises from the product in the frequency domain.

This relation is valid for $2 \mathrm{D}$ and $3 \mathrm{D}$ space. The decorrelation $D C$ is proportional to the total cross-section of the new defect $\sigma$ and the wave velocity $c$. It also depends on the position of the defect $\boldsymbol{r}_{\mathbf{0}}$ and the coda time $t$ through the sensitivity kernel $K$ :

$$
\begin{aligned}
D C\left(s, \boldsymbol{r}, \boldsymbol{r}_{\mathbf{0}}, t\right) & =\frac{c \sigma}{2} K\left(\boldsymbol{s}, \boldsymbol{r}, \boldsymbol{r}_{\mathbf{0}}, t\right), \\
K\left(\boldsymbol{s}, \boldsymbol{r}, \boldsymbol{r}_{\mathbf{0}}, t\right) & =\frac{\int_{0}^{t} I\left(\boldsymbol{s}, \boldsymbol{r}_{\mathbf{0}}, u\right) I\left(\boldsymbol{r}_{\mathbf{0}}, \boldsymbol{r}, t-u\right) d u}{I(\boldsymbol{s}, \boldsymbol{r}, t)}
\end{aligned}
$$


This kernel was first introduced by Pacheco and Snieder [24] to describe the time-shift of scattered waves induced by a local velocity change in a heterogeneous medium. Here, we demonstrated that it also allows to describe the decorrelation induced by a local structural change. It is also important to note that the kernel does not depend on the absorption in the medium, as long as it is spatially uniform.

In the next subsection, using the formalism developed above, we study the time shift induced by a slight velocity change.

\subsection{Estimation of the time-shift induced by a weak velocity change}

In the case of a weak but global velocity change, i.e. occurring all over the medium, a simple relation describes the time-shift of diffuse waves:

$$
\begin{aligned}
\frac{\delta t}{t} & =-\frac{\delta c}{c} \\
\Leftrightarrow \delta t(t) & =-\frac{\delta c}{c} t
\end{aligned}
$$

The time-shift $\delta t(t)$ induced by the global relative velocity change $\delta c / c$ grows linearly with coda time $t$ (nb: this relation could be inaccurate in the case of resonant scattering because the time spent by the waves inside the scatterers may depend critically on $\delta c / c$ [32]).

In the case of a local change, just a small part of the multiply scattered waves will propagate through the velocity variation area and the above relation does not apply anymore. In addition to the time shift, we also expect a distortion or decorrelation of the waveforms. We will show in the following that the latter effect is small, making the study of the time shift more relevant.

Using the notations of the previous section, we introduce a cross-correlation function $w$ :

$$
\bar{w}(t, \tau, \boldsymbol{s}, \boldsymbol{r})=\overline{g_{2}\left(\boldsymbol{s}, \boldsymbol{r}, t+\frac{\tau}{2}\right) g_{1}^{*}\left(\boldsymbol{s}, \boldsymbol{r}, t-\frac{\tau}{2}\right)}
$$

where $\tau$ is the phase-shift between the initial and final Green's functions and where the overline still designate the ensemble average operation. By expanding the Green's functions over their frequency components, we express the cross-correlation function as:

$$
\bar{w}(t, \tau)=\frac{1}{(2 \pi)^{2}} \iint \overline{G_{2}^{+}\left(\boldsymbol{s}, \boldsymbol{r}, \omega+\frac{\Omega}{2}\right) G_{1}^{-}\left(\boldsymbol{s}, \boldsymbol{r}, \omega-\frac{\Omega}{2}\right)} e^{-i \omega \tau} e^{-i \Omega t} d \Omega d \omega
$$

where $G_{2}^{+}$(resp. $G_{1}^{-}$) corresponds to the retarded (resp. advanced) Green's function in the final (resp. initial) state, in the frequency domain. $\omega$ is the conjugate variable of the time shift $\tau$ that describes the fast variations of the wavefield and $\Omega$ is the conjugate variable of time $t$ that describes the slow variations of the ensemble averaged correlation. Here, we aim to determine the optimal time shift value $\tau_{\text {opt }}(t)$ that maximizes the correlation $\bar{w}(t, \tau)$. We thus derivate the correlation (23) with respect to $\tau$ to obtain:

$$
\iint \overline{G_{2}^{+}\left(\boldsymbol{s}, \boldsymbol{r}, \omega+\frac{\Omega}{2}\right) G_{1}^{-}\left(\boldsymbol{s}, \boldsymbol{r}, \omega-\frac{\Omega}{2}\right)} e^{-i \Omega t} d \Omega \omega e^{-i \omega \tau_{\mathrm{opt}}} d \omega=0
$$

As in the previous subsection, calculating the correlation term $\overline{G_{2}^{+} G_{1}^{-}}$involves expanding the initial and final Green's functions. To start with, let us suppose that the change is 
small enough to be considered point-like. We then use the expansion of the correlation term $G_{2}^{+} G_{1}^{-}$derived in the previous subsection for a point scatterer at $\boldsymbol{r}_{\mathbf{0}}(14)$ :

$$
\begin{aligned}
\overline{G_{2}^{+}(\boldsymbol{s}, \boldsymbol{r}) G_{1}^{-}(\boldsymbol{s}, \boldsymbol{r})} & =I_{11}^{2} L_{\Omega}(\boldsymbol{s}, \boldsymbol{r})+I_{11}^{2} I_{21}(\omega) \mathfrak{t}_{0}(\omega) L_{\Omega}\left(\boldsymbol{s}, \boldsymbol{r}_{\mathbf{0}}\right) L_{\Omega}\left(\boldsymbol{r}_{\mathbf{0}}, \boldsymbol{r}\right) \\
& =\frac{c I_{11}}{\ell} I_{\Omega}(\boldsymbol{s}, \boldsymbol{r})+\frac{c^{2} I_{21}(\omega)}{\ell^{2}} \mathfrak{t}_{0}(\omega) I_{\Omega}\left(\boldsymbol{s}, \boldsymbol{r}_{\mathbf{0}}\right) I_{\Omega}\left(\boldsymbol{r}_{\mathbf{0}}, \boldsymbol{r}\right)
\end{aligned}
$$

where we also used the relation between the Ladder $L_{\Omega}$ and the normalized diffusive intensity $I_{\Omega}$ (A9). We remind that $\mathfrak{t}_{0}(\omega)$ represents the $\boldsymbol{T}$-matrix of the point defect located in $\boldsymbol{r}_{\mathbf{0}}, L_{\Omega}$ is the Ladder operator that describes all the scattering events between two positions and $I_{\Omega}$ is the normalized diffusive intensity in the frequency domain. The loop integrals $I_{11}$ and $I_{21}(\omega)$ are calculated in the Appendix 2. We apply the inverse Fourier transform over $\Omega$ :

$$
\begin{aligned}
& \int \overline{G_{2}^{+}(\boldsymbol{s}, \boldsymbol{r}) G_{1}^{-}(\boldsymbol{s}, \boldsymbol{r})} e^{-i \Omega t} d \Omega \propto I_{11} I(\boldsymbol{s}, \boldsymbol{r}, t) \\
& \quad+\frac{c I_{21}(\omega)}{\ell} \mathfrak{t}_{\mathcal{o}}(\omega) \int_{0}^{t} I\left(\boldsymbol{s}, \boldsymbol{r}_{\mathbf{0}}, u\right) I\left(\boldsymbol{r}_{\mathbf{0}}, \boldsymbol{r}, t-u\right) d u
\end{aligned}
$$

And we now insert expression (26) into (24) to obtain:

$$
\begin{aligned}
I_{11} I(\boldsymbol{s}, \boldsymbol{r}, t) \int \omega e^{-i \omega \tau_{\mathrm{opt}}} d \omega= & -\frac{c}{\ell} \int_{0}^{t} I\left(\boldsymbol{s}, \boldsymbol{r}_{\mathbf{0}}, u\right) I\left(\boldsymbol{r}_{\mathbf{0}}, \boldsymbol{r}, t-u\right) d u \\
& \times \int \omega I_{21}(\omega) \mathbf{t}_{\mathbf{0}}(\omega) e^{-i \omega \tau_{\mathrm{opt}}} d \omega
\end{aligned}
$$

Considering signals that are filtered in a narrow frequency band $\Delta \omega$ around $\omega_{0}$ and that the time-shift induced by the weak velocity change is much smaller than the central period ( $\left.\tau_{\mathrm{opt}} \ll 2 \pi / \omega_{0}\right)$, we approximate $e^{-i \omega \tau_{\mathrm{opt}}}$ as $1-i \omega \tau_{\mathrm{opt}}$. Using the hermitian symmetry of $I_{21}(\omega)(\mathrm{B} 13, \mathrm{~B} 18)$ and $\mathfrak{t}(\omega)(10)$, we calculate the integrals of Equation (27):

$$
\begin{gathered}
\int \omega e^{-i \omega \tau_{\mathrm{opt}}} d \omega \simeq-i \tau_{\mathrm{opt}} \omega_{0}^{2} \Delta_{\omega} \\
\int \omega I_{21}(\omega) \mathfrak{t}_{\mathcal{o}}(\omega) e^{-i \omega \tau_{\mathrm{opt}}} d \omega \simeq \delta_{\omega} I_{21} \omega_{0}\left(\Re\left(\mathfrak{t}_{\mathfrak{o}}\right)+\tau_{\mathrm{opt}} \omega_{0} \Im\left(\mathfrak{t}_{\mathfrak{o}}\right)\right)
\end{gathered}
$$

The defect being a weak velocity change, its $\boldsymbol{T}$-matrix is calculated within the first-order Born approximation:

$$
\begin{aligned}
\mathfrak{t}_{\mathrm{o}} & =V\left(\boldsymbol{r}_{\mathbf{0}}\right) v_{s} \\
& =v_{s} \omega_{0}^{2}\left(\frac{1}{c^{2}}-\frac{1}{c\left(r_{0}\right)^{2}}\right) \\
& \simeq 2 v_{s} k_{0}^{2} \frac{\delta c}{c}
\end{aligned}
$$

where $V\left(\boldsymbol{r}_{\mathbf{0}}\right)$ is the potential of the defect. The wave speed in the defect of volume $v_{s}$ reads $c\left(r_{0}\right)=c+\delta c$, with $c$ the background velocity, and assuming $\delta c \ll c$. In the Born approximation, the $\boldsymbol{T}$-matrix of the defect is real $\left(\Im\left(\mathfrak{t}_{\mathfrak{o}}\right)=0\right)$, which implies that a really low decorrelation is expected (16). Equation (27) simplifies to: 


$$
\tau_{\mathrm{opt}}=\frac{I_{21}}{i I_{11} k_{0} \ell} \mathfrak{t}_{\mathrm{o}} \frac{\int_{0}^{t} I\left(\boldsymbol{s}, \boldsymbol{r}_{\mathbf{0}}, u\right) I\left(\boldsymbol{r}_{\mathbf{0}}, \boldsymbol{r}, t-u\right) d u}{I(\boldsymbol{s}, \boldsymbol{r}, t)}
$$

The ratio of the loop integrals is $\frac{I_{21}}{I_{11}}=-\frac{i \ell}{2 k}$ in $2 \mathrm{D}$ and $3 \mathrm{D}$ (Appendix 2). Using also the approximation of the $\boldsymbol{T}$-matrix (30), we finally obtain:

$$
\tau_{\mathrm{opt}}(t)=-\frac{\delta c}{c} v_{s} \frac{\int_{0}^{t} I\left(\boldsymbol{s}, \boldsymbol{r}_{\mathbf{0}}, u\right) I\left(\boldsymbol{r}_{\mathbf{0}}, \boldsymbol{r}, t-u\right) d u}{I(\boldsymbol{s}, \boldsymbol{r}, t)}
$$

where we recognize the sensitivity kernel $K\left(\boldsymbol{s}, \boldsymbol{r}, \boldsymbol{r}_{\mathbf{0}}, t\right)$ introduced in the previous subsection (20), for a source at $\boldsymbol{s}$, a receiver at $\boldsymbol{r}$, a change at $\boldsymbol{r}_{\mathbf{0}}$, and a measurement around time $t$ in the coda.

In this situation of a local weak velocity change, the time shift $\tau$ is proportional to the relative velocity change in the medium $\delta c / c$ and to the scatterer's volume $v_{s}$ but does not grow linearly with coda time. In practice, this is a way to discriminate a global velocity change from a local velocity change. A numerical study of a velocity change introduced in a layer at depth is performed by Obermann et al. [33].

We can now address the case of an extended weak velocity change. Contrary to the case of the decorrelation induced by a structural change (strong impedance contrast), here, the Born approximation allows us to describe the time shift $\tau$ associated to a spatially extended velocity change. We insist on the fact that the Born approximation is only valid for the change but not for the underlying multiple scattering medium. In this approximation, the correlation term is written as:

$$
\overline{G_{2}^{+}(\boldsymbol{s}, \boldsymbol{r}) G_{1}^{-}(\boldsymbol{s}, \boldsymbol{r})}=I_{11}^{2} L_{\Omega}(\boldsymbol{s}, \boldsymbol{r})+I_{11}^{2} I_{21}(\omega) \int L_{\Omega}\left(\boldsymbol{s}, \boldsymbol{r}_{\mathbf{0}}\right) V\left(\boldsymbol{r}_{\mathbf{0}}\right) L_{\Omega}\left(\boldsymbol{r}_{\mathbf{0}}, \boldsymbol{r}\right) d \boldsymbol{r}_{\mathbf{0}}
$$

which is a generalization of expression (25) in the case of an extended defect. This formulation is only possible because we replaced the $\boldsymbol{T}$-matrix of the extended defect by its potential that only depends on one spatial variable. It is not applicable in the general case where the $\boldsymbol{T}$-matrix of an extended defect would depend on two spatial variables.

The potential $V$ of the extended defect reads $V\left(\boldsymbol{r}_{\mathbf{0}}\right) \simeq 2 k^{2} \frac{\delta c}{c}\left(\boldsymbol{r}_{\mathbf{0}}\right)$, similarly to (30), but now the relative velocity variation depends on the position $\boldsymbol{r}_{\mathbf{0}}$ in the studied medium. The intermediate calculation steps apply identically and the generalized result reads:

$$
\tau_{\mathrm{opt}}(t)=-\int_{\mathrm{Vol}} \frac{\delta c}{c}\left(\boldsymbol{r}_{\mathbf{0}}\right) K\left(\boldsymbol{s}, \boldsymbol{r}, \boldsymbol{r}_{\mathbf{0}}, t\right) d \boldsymbol{r}_{\mathbf{0}}
$$

where the integral covers all the studied medium and where the relative velocity variation $\frac{\delta c}{c}$ is null outside the change. This formulation is identical to the one derived by Pacheco and Snieder [24] with a random walk approach.

If the relative velocity change occurs in the whole medium, we obtain:

$$
\tau_{\mathrm{opt}}(t)=-\frac{\delta c}{c} \int_{\mathrm{Vol}} K\left(\boldsymbol{s}, \boldsymbol{r}, \boldsymbol{r}_{\mathbf{0}}, t\right) d \boldsymbol{r}_{\mathbf{0}}
$$

The spatial integral of kernel $\int_{\mathrm{Vol}} K\left(\boldsymbol{s}, \boldsymbol{r}, \boldsymbol{r}_{\mathbf{0}}, t\right) d \boldsymbol{r}_{\mathbf{0}}=t$ is evaluated using the identity $\int_{\mathrm{Vol}} I\left(\boldsymbol{s}, \boldsymbol{r}_{\mathbf{0}}, u\right) I\left(\boldsymbol{r}_{\mathbf{0}}, \boldsymbol{r}, t-u\right) d \boldsymbol{r}_{\mathbf{0}}=I(\boldsymbol{s}, \boldsymbol{r}, t)$, which is a special formulation of the Chapman-Kolmogorov equation [34]. This property is also stressed in Pacheco and Snieder [24]. It allows to understand the kernel $K\left(\boldsymbol{s}, \boldsymbol{r}, \boldsymbol{r}_{\mathbf{0}}, t\right)$ as a volume density of time (also called local time [35]) spent around $\boldsymbol{r}_{\mathbf{0}}$ by waves that have traveled from $\boldsymbol{s}$ to $\boldsymbol{r}$ during time $t$. The 
expression (35) then simplifies to $\tau_{\mathrm{opt}}(t)=-\frac{\delta c}{c} t$. We recover the identity (21) introduced at the beginning of the subsection to characterize the time shift induced by a global velocity change.

Let us now study in more details the spatial and temporal behavior of the sensitivity kernel $K(20)$.

\section{Sensitivity Kernels in two and three dimensions}

Using the diffusion solution as the intensity propagator, the sensitivity kernel (20) has analytical solutions in 2D and 3D infinite media.[35] These expressions and their representations are gathered in Section 4.1. The derivation of the theoretical decorrelation and time-shift in Section 3 has been done within the diffusive regime; however, it is reasonable to assume that the expression of the sensitivity kernel (20) remains valid in more general transport regimes. In Section 4.2, we build sensitivity kernels based on the radiative transfer approximation and we compare these new kernels (2D and 3D) with the diffusion-based ones.

\subsection{Kernels in the diffusion approximation}

Using the infinite medium diffusion solution (A10), the kernel (20) has analytical expressions in $2 \mathrm{D}$ and $3 \mathrm{D}[35]$ :

$$
\begin{gathered}
K_{3 D}(a, b, R, t)=\frac{a+b}{4 \pi D a b} e^{\frac{R^{2}-(a+b)^{2}}{4 D t}} \\
K_{2 D}(a, b, R, t)=\frac{1}{2 \pi D} e^{\frac{R^{2}-\left(a^{2}+b^{2}\right)}{4 D t}} K_{0}\left(\frac{a b}{2 D t}\right)
\end{gathered}
$$

where $a=\left\|\boldsymbol{s}-\boldsymbol{r}_{\mathbf{0}}\right\|$ is the source-defect distance, $b=\left\|\boldsymbol{r}-\boldsymbol{r}_{\mathbf{0}}\right\|$ the defect-receiver distance, and $R=\|\boldsymbol{s}-\boldsymbol{r}\|$ the source-receiver distance. $K_{0}$ is the modified Bessel function of the second kind. We remind that the kernels do not depend on the absorption of the medium, as long as it is spatially uniform.

These kernels are plotted in Figure 4 as a function of the position of the defect, for a fixed source-receiver separation. The two peaks of sensitivity correspond to the position of the source and receiver, meaning that the decorrelation (or phase-shift) is higher when the change is occurring near these points. The sensitivity is also higher along the sourcereceiver segment than elsewhere in the medium. Due to the diffusive nature of the energy propagation, the spatial extent $L$ of the peaks is governed by the Thouless time $L^{2} / D$. This implies that the kernel keeps a strong spatial gradient even at large times in the coda. For imaging purposes, measurements should be performed at different times in the coda to obtain a good spatial resolution all over the studied medium.

\subsection{Kernels in the radiative transfer approximation}

The diffusion approximation for the intensity transport is only valid at large times compared to the mean free time $(t \gg \ell / c)$. Since the kernel involves a temporal convolution in its numerator, its expression may be valid only when the defect is located far enough 

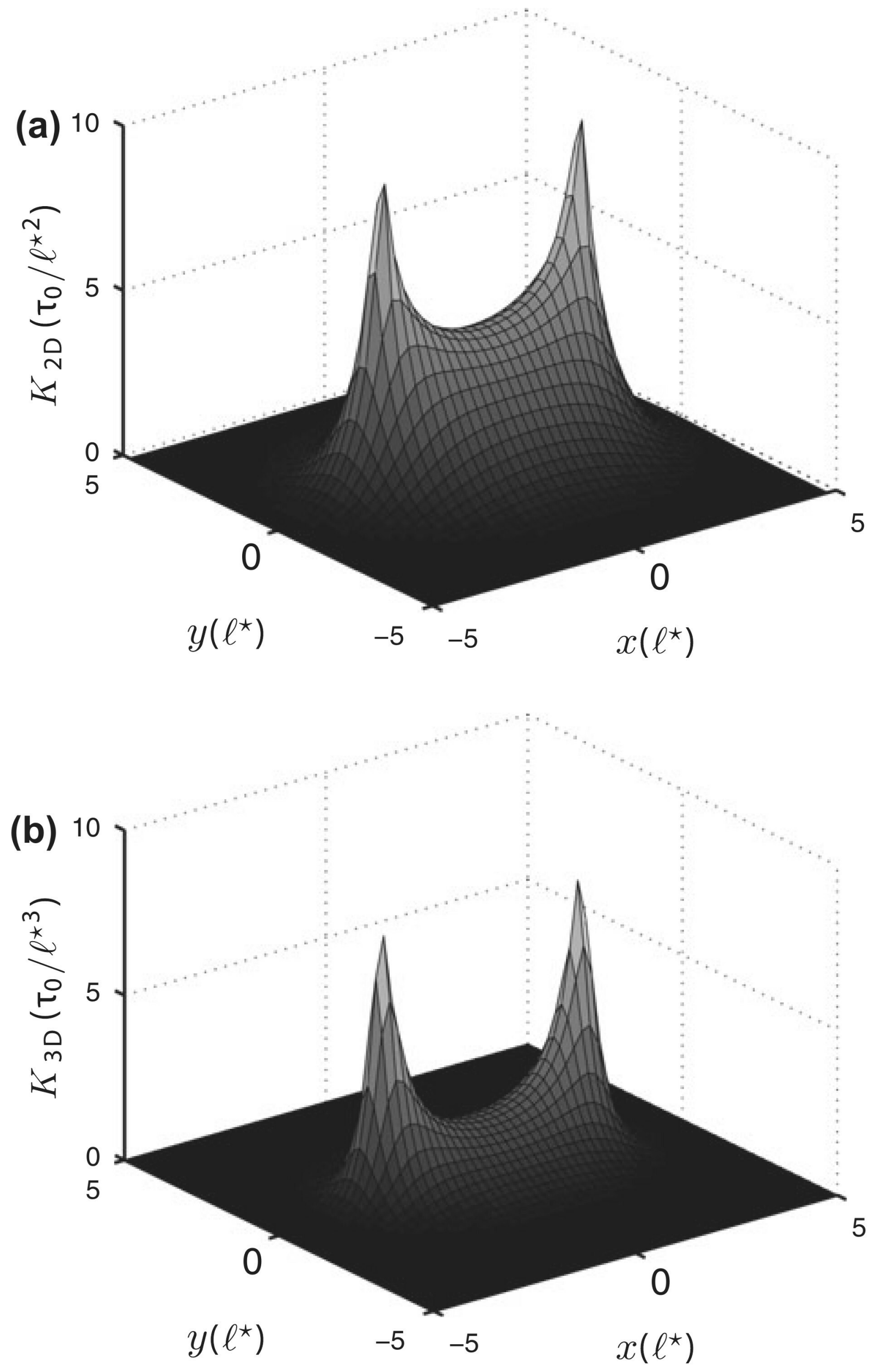

Figure 4. Diffusion-based sensitivity kernels in 2D (a) and 3D (b) vs. the position of the defect $(x, y)$. The source-receiver distance is fixed at $R=5 \ell^{\star}$ and the measurement time at $t=10 \tau, \tau=\ell^{\star} / c$ being the transport mean free time. In $3 \mathrm{D}$, for a better visualization, the source and receiver are kept at a distance $z=\ell^{\star} / 4$ from the observation plan.

from the source and receiver $(a, b \gg \ell)$. A more accurate way to describe the intensity transport in the ISA is to solve the radiative transfer equation. This equation has been first introduced phenomenologically by Chandrasekhar [36]. For a detailed review of its analytical derivation, the reader might refer to Lagendijk and Van Tiggelen [37] as a general reference and to Margerin [38] for seismological applications.

In the case of isotropic scattering, the radiative transfer equation has an analytical solution in 2D infinite media. This solution has been first derived by Shang and Gao [39], and later by Sato [40] and Paasschens [41]. The expression for the intensity reads:

$$
I_{2 \mathrm{DT}}^{\mathrm{RT}}(r, t)=\frac{e^{-\frac{r}{\ell}}}{2 \pi r c} \delta\left(t-\frac{r}{c}\right)+\frac{1}{4 \pi D t}\left(1-\frac{r^{2}}{c^{2} t^{2}}\right)^{-\frac{1}{2}} e^{\frac{1}{\ell}\left(\sqrt{c^{2} t^{2}-r^{2}}-c t\right)} \Theta(c t-r)
$$


where the isotropic scattering implies the equality of the scattering and transport mean free paths $\ell=\ell^{\star}$. The diffusion constant is $D=c \ell^{\star} / 2$ and the Heaviside function $\Theta$ ensures the causality of the solution (the energy cannot travel faster than the wave velocity).

This solution is composed of the coherent intensity term, decaying exponentially with the mean free path and of a diffuse term, that includes all the scattering orders. It is thus also valid at short times and distances and one can verify that this solution converges towards the diffusion solution for times $t \gg \ell / c$.

In 3D, an approximate solution has been derived by Paasschens [41] as an interpolation of the 2D and 4D analytical solution. In both cases, the radiative transfer intensity can be written as a sum of coherent $I^{c}$ and incoherent $I^{i}$ intensity:

$$
I^{\mathrm{TR}}=I^{c}+I^{i}
$$

Let us suppose that the general form of the sensitivity kernel (20) remains valid in the radiative transfer approximation. We then replace the diffusion solution intensity by the radiative transfer solution intensity. Using the above expression (39), the convolution in the numerator $n(a, b, t)$ of the kernel $K$ can be decomposed into four terms:

$$
\begin{aligned}
K(a, b, R, t)= & \frac{n(a, b, t)}{I^{\mathrm{RT}}(R, t)}, \\
n(a, b, t)= & \int_{0}^{t} I^{c}(a, u) I^{c}(b, t-u) d u\left(n^{c c}\right) \\
& +\int_{0}^{t} I^{c}(a, u) I^{i}(b, t-u) d u\left(n^{c i}\right) \\
& +\int_{0}^{t} I^{i}(a, u) I^{c}(b, t-u) d u\left(n^{i c}\right) \\
& +\int_{0}^{t} I^{i}(a, u) I^{i}(b, t-u) d u\left(n^{i i}\right)
\end{aligned}
$$

The first term $n^{c c}$ arises from the convolution of the coherent wave propagating from the source to the defect and the coherent wave propagating from the defect to the receiver. This term is non-zero only at time $t=\frac{a+b}{c}$ corresponding to the single scattered path (source-defect-receiver). We neglect this term, since we focus on coda waves.

The second term $n^{c i}$ (resp. third term $n^{i c}$ ) is a crossed term, that corresponds to a coherent (resp. incoherent) propagation from the source to the defect and an incoherent (resp. coherent) propagation from the defect to the receiver. This term becomes important if the defect is close to the source (resp. the receiver).

The fourth and last term $n^{i i}$ corresponds to an incoherent propagation from the source to the defect and also incoherent from the defect to the receiver. We will observe that this term converges towards the diffusion-based kernel at long times.

The second and third terms have analytical expressions thanks to the Dirac delta function in the coherent intensity. The fourth term may be evaluated numerically. Slices of the radiative transfer-based kernels are plotted in Figure 5. For comparison, the diffusion-based kernels are also represented.

The kernels in the radiative transfer and diffusion approximation have similar shapes. However, one can notice a difference of sensitivity close to the source and receiver. This difference is particularly significant in an area of radius $\approx \ell$ around these points. The extra sensitivity is mainly due to the crossed terms $\left(n^{c i}\right.$ and $\left.n^{i c}\right)$ that describe the interaction of 

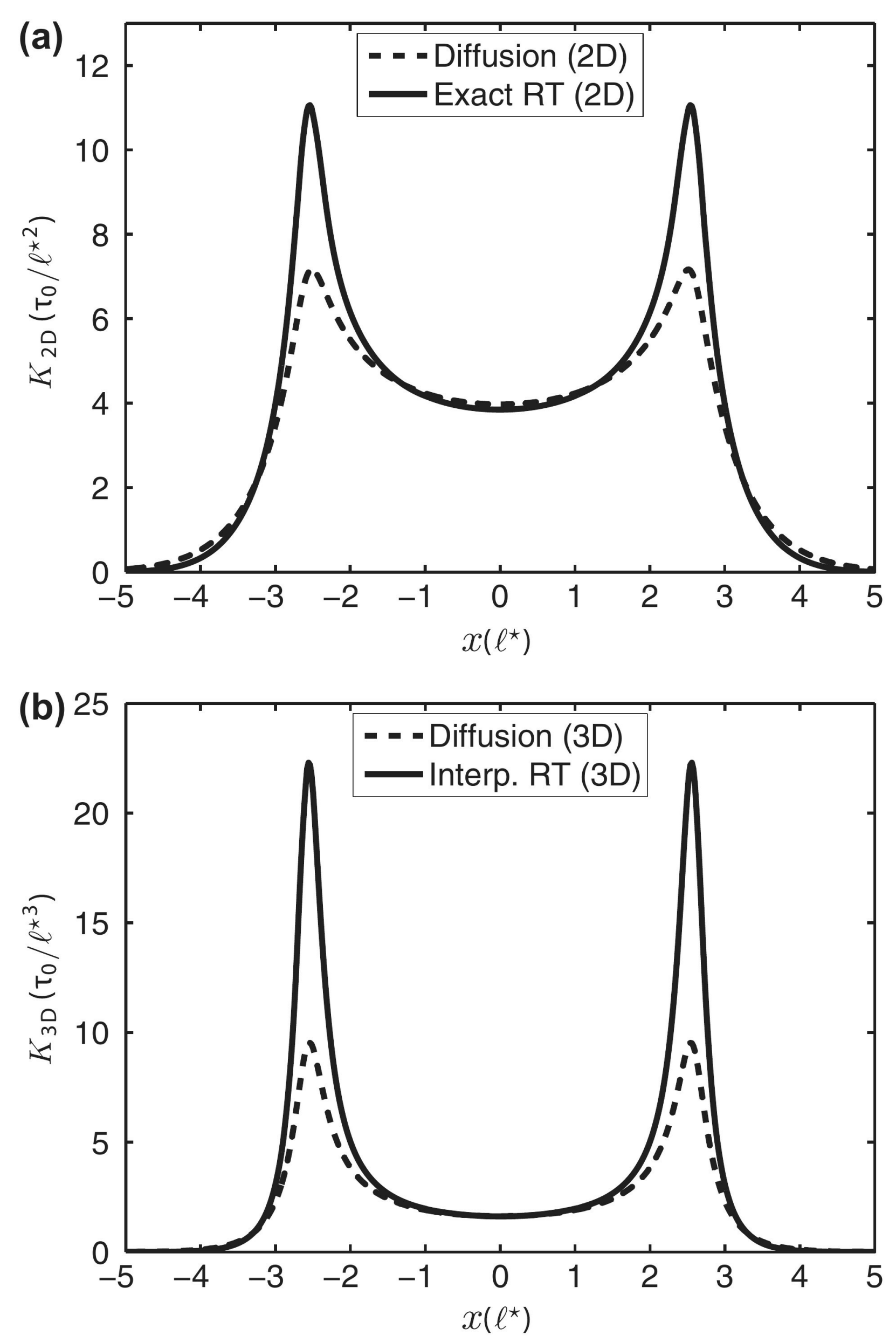

Figure 5. Radiative transfer (solid line) and diffusion (dashed line) based kernels, in 2D (a) and 3D (b). The source-receiver distance is $R=5 \ell^{\star}$ and the time $t=10 \tau, \tau=\ell^{\star} / c$ being the transport mean free time. The spatial dependence of the kernel is represented for defects located along an axis parallel to the source-receiver axis at an offset of $y=\ell^{\star} / 5$.

the coherent wave with the defect. The extension of this area corresponds to the typical decay length of the coherent wave.

The temporal dynamics of the kernels will be examined in the next section, and tested with measurements of decorrelation from numerical simulations.

\section{Numerical validation}

In this section, through a series of 2D numerical simulations introduced in Section 2, we validate the theoretical approach presented in Section 3.1. We perform our simulations in lossless media, but we remind that any spatially uniform absorption has no influence on the present study. Between two states, a new scatterer is introduced in the heterogeneous medium. We then compare in different configurations the resulting decorrelation of the sim- 


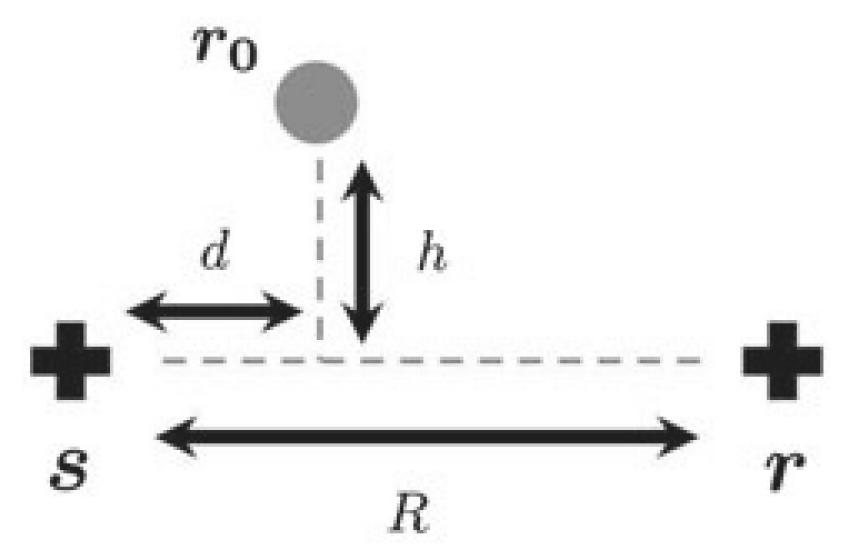

Figure 6. Schematic representation of the source, receiver, and defect positions.

ulated waveforms with the theoretical predictions based on the sensitivity kernels developed in Section 4. In the following tests, the source-receiver distance is fixed $\left(R=8 \ell^{\star}\right)$ and the position of the new scatterer is defined by the variables $d$ and $h$ as illustrated in Figure 6 .

The decorrelation is measured in a moving window of length $T \simeq 50 \tau_{0}$ along the time $t$ in the coda. To reduce the fluctuations of the measurements, we also average each decorrelation over 50 different realizations of the disorder (i.e. background distribution of the scatterers in the medium). This operation also allows to estimate the standard deviation of the measurements. We emphasize that we average these measurements only to help us demonstrate the validity of our model. For imaging purposes, these operations are not necessary and are compensated by the use of several sources and receivers in the medium.

The theoretical decorrelation is computed both in the diffusion and radiative transfer approximation. To take into account the effect of the reflective boundaries, we use the method of images.[42] The total intensity is written as the sum of the intensity of the real source in an infinite medium plus the contribution of the intensity of virtual mirror images.

We choose to simulate reflective boundary conditions because they often correspond to real life cases, in non-destructive testing of materials for instance. In the case of changes occuring in infinite media, the expression of the decorrelation would be directly related to the infinite medium kernels studied in Section 4.

We here consider the decorrelation induced by a point scatterer appearing at different positions in the medium. This new scatterer is identical to the thousand ones already present in the medium. Its scattering cross-section is known $\left(\sigma \simeq 0.174 \lambda_{0}\right)$ and we thus compute the theoretical decorrelation (19) without adjusting any parameter.

\subsection{Distant change}

In this first experiment, we place the defect far away from the source and receiver by choosing $d=4 \ell^{\star}$ (see Figure 6). We then study the influence of the distance $h$ between the defect and the source-receiver axis (from $h=0$ to $h=8 \ell^{\star}$ ). In any case, the propagation distance is larger than a mean free path. The decorrelations measured as a function of the time in the coda (and averaged over the disorder) are represented by crosses in Figure 7. The diffusion-based decorrelations are represented by dotted lines and the radiative transferbased decorrelations by solid lines.

The measured decorrelation is well described by the two models, which rapidly converge towards each other. However, at short times, we notice the slight inaccuracy of the diffusionbased kernel which overestimates the decorrelation. This effect is due to the instantaneous transport of the energy in the diffusion approximation (not limited by the wave velocity). 


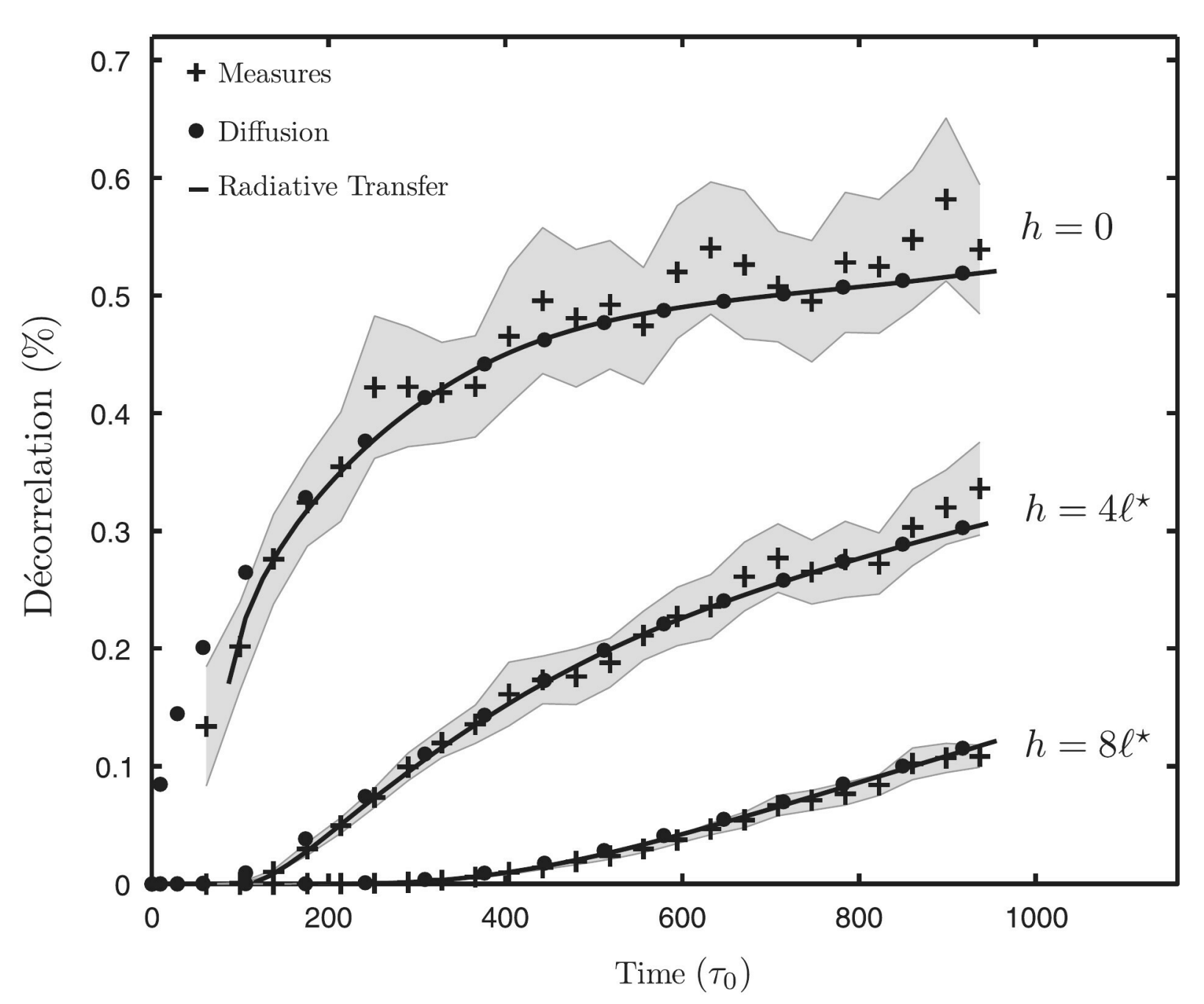

Figure 7. Decorrelation induced by a distant point defect: $d=4 \ell^{\star}$ and $h=0,4,8 \ell^{\star}$. We represent the diffusion-based decorrelation (dots), the radiative transfer-based decorrelation (solid lines), and the decorrelation measured from the simulations (crosses). The shaded areas delimit the \pm 1 standard deviation uncertainties.

\subsection{Close change}

In a second set of experiments, we study the decorrelation induced by a scatterer appearing close to the source $\left(d=\ell^{\star} / 2, \ell^{\star} / 4\right)$. We place the defect on the source-receiver axis $(h=0)$, and keep the source-receiver distance $R=8 \ell^{\star}$ as before. The decorrelations measured as a function of time in the coda (and averaged over the disorder) are represented by crosses in Figure 8 . The diffusion-based decorrelations are represented by dotted lines and the radiative transfer-based decorrelations by solid lines.

In this case, the diffusion-based kernel is inaccurate whatever the time in the coda. This systematic error is due to the omission of the coherent wave propagating from the source to the defect. Indeed, when it is located at less than one mean free path from the source, a non-negligible part of the decorrelation comes from the interaction of the coherent wave with the defect. This contribution is well described by the kernel obtained in the radiative transfer approximation.

To conclude, the diffusion-based kernel can only be used to describe the decorrelation induced by defects appearing far away from the source and receiver (a few $\ell$ ), and at large times in the coda. The radiative transfer-based kernel seems much more robust and can describe the decorrelation whatever the time in the coda and the position of the defect.

We here considered the case of isotropic scattering in the background medium, for which the radiative transfer equation has an analytical solution (38). In the anisotropic scattering case, one can use numerical solutions of the radiative transfer equation to build the sensitivity kernel. As a proof of feasibility, we build a sensitivity kernel based on a Monte-Carlo simulated solution of the radiative transfer equation (Figure 8). Even if we did it within our isotropic scattering case, this example let us foresee the possibility to monitor more general media. 


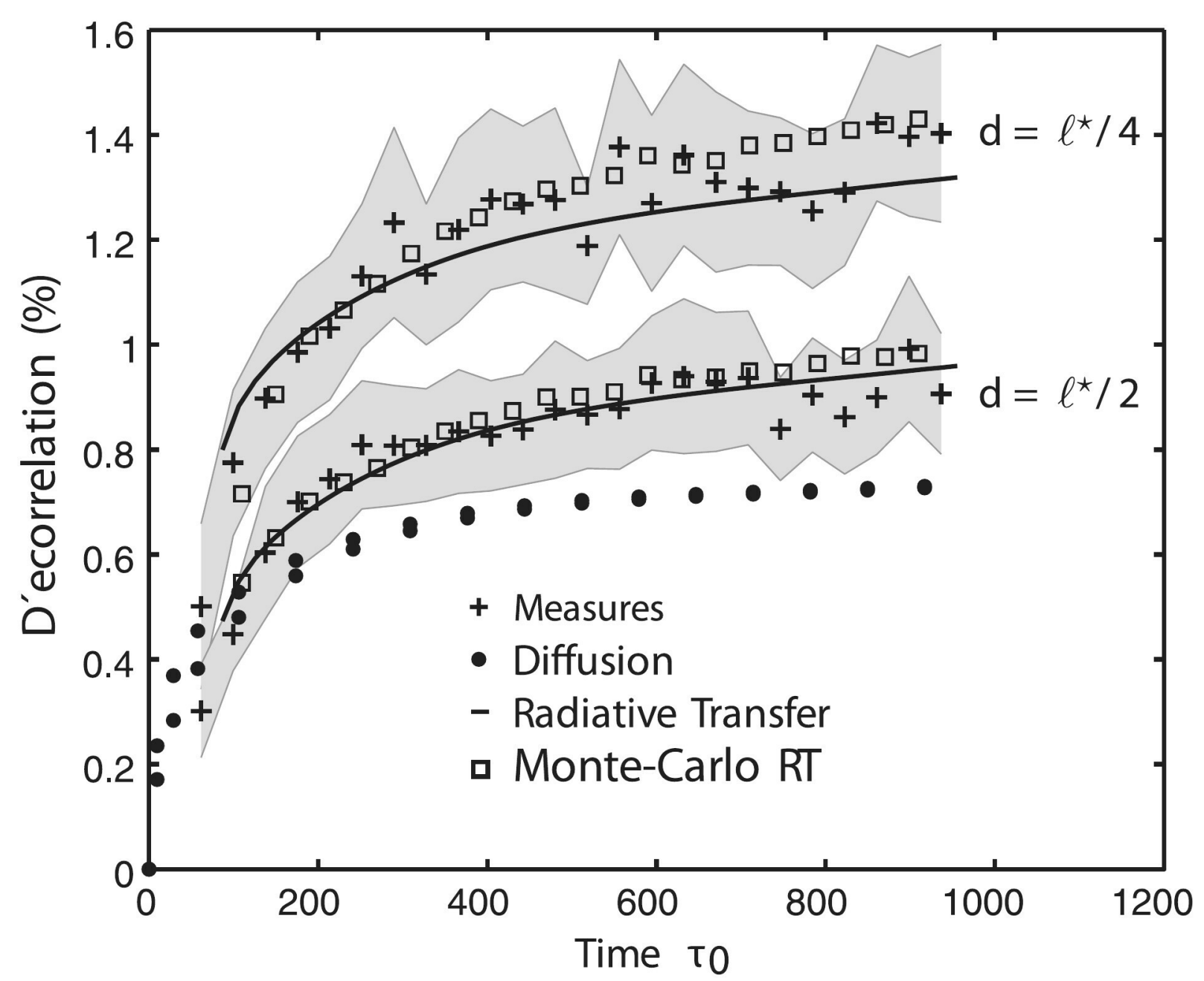

Figure 8. Decorrelation induced by a close point defect: $d=0.25,0.5 \ell^{\star}$ and $h=0$. We represent the diffusion-based decorrelation (dots), the exact radiative transfer-based decorrelation (solid lines), the Monte-Carlo radiative transfer-based decorrelation (squares), and the decorrelation measured from the simulations (crosses). The shaded areas delimit the \pm 1 standard deviation uncertainties.

We also restricted our numerical experiments to point-like changes. From a theoretical point of view, the decorrelation induced by a finite-size scatterer has yet to be investigated. The main issue comes from the expression of the $\boldsymbol{T}$-matrix of a finite-size scatterer, which is not diagonal. In simpler terms, that means that we have to take into account paths that visit the new scatterer several times, at different locations. This complication is avoided in the case of the extended velocity change because of the Born approximation applied to the change. We remind that this approximation allows to replace the $\boldsymbol{T}$-matrix of the change by its potential, which is a diagonal operator.

\section{Conclusion}

We studied the decorrelation and phase-shift of coda waves induced by local changes in heterogeneous media. Using the multiple scattering formalism, we derived an analytical expression for the decorrelation induced by a point-like structural change (strong impedance contrast). In the case of a slight velocity change (weak impedance contrast), we derived an analytical expression for the phase-shift induced on coda waves. Contrary to the decorrelation, this time-lapse can be evaluated for a spatially extended change, and we recovered analytically an expression that was previously introduced in a probabilistic sense.[24]

The expression of the decorrelation and time-shift are both related to a same sensitivity kernel, based on the intensity transport in the medium. The kernel depends on the position of the source and receiver, the position of the change, and the time in the coda. The decorrelation also depends on the scattering cross-section of the change while the phase-shift depends on the relative velocity variation of the change.

We derived these expressions within the diffusive regime, where the diffusion-based sensitivity kernels have analytical forms in 2D and 3D infinite media. We also suppose that the kernel formulation remains valid outside this regime, using the appropriate intensity 
propagator. We numerically evaluated the expression of the sensitivity kernel based on the radiative transfer solution, for $2 \mathrm{D}$ and $3 \mathrm{D}$ infinite media, in the case of isotropic scattering. Comparing these kernels with the diffusion-based ones, we observed that the differences in sensitivity are localized in a region of a mean free path $\ell$ around the source and the receiver.

To validate the theoretical estimation of the decorrelation, we performed a series of numerical simulations of acoustic waves propagating in 2D heterogeneous media. To simulate the apparition of a local change, we added a point scatterer at different positions in the medium. We then compared the decorrelation measured on the waveforms with the ones calculated with the sensitivity kernels in both the diffusion and the radiative transfer approximation. The diffusion-based kernel allows to describe the decorrelation at large times in the coda and when the change is located far away from the source and receiver (a few $\ell$ ). However, to describe the decorrelation at short times in the coda or when the change is located near the source or receiver, it is necessary to use the radiative transferbased kernel. This is understood regarding the validity domain of the diffusion and radiative transfer solutions. The diffusion solution accurately describes the intensity transport only at large times compared to the mean free time. The radiative transfer solution, that also includes the intensity of the coherent wave and low order scattering, is accurate at all times.

This study brings new insights on the information carried by coda waves. Despite their random appearance and the complexity of the trajectories, the variations they undergo are strongly related to the position and strength of the changes in the medium. These dependencies have recently been used for imaging purposes as in a non-destructive testing of concrete experiment [25] and in the monitoring of temporal changes in the earth's crust.[27,28,33]

The results presented in this article give a general and common theoretical background of these imaging techniques, and point out possible future improvements (as the use of radiative transfer based kernels).

\section{Acknowledgements}

C.S. acknowledges support by the BMBF GeoRechnologien Program (FKZ 03G0736A).

\section{Funding}

This work was funded by the ANR [grant number JC08_313906 SISDIF], and by the VOR program.

\section{References}

[1] Derode A, Tourin A, Fink M. Random multiple scattering of ultrasound. i. coherent and ballistic waves. Phys. Rev. E. 2001;64:036605.

[2] Anugonda P, Wiehn J, Turner J. Diffusion of ultrasound in concrete. Ultrasonics. 2001;39:429435.

[3] Ryzhik L, Papanicolaou G, Keller JB. Transport equation for elastic and other waves in random media. Wave Motion. 1996;24:327-370.

[4] Aki K, Chouet B. Origin of coda waves: source, attenuation and scattering effects. J. Geophys. Res. 1975;80:3322-3342.

[5] Albada M, Lagendijk A. Observation of weak localization of light in a random medium. Phys. Rev. E. 1985;55:2692-2695. 
[6] Wolf P, Maret G. Weak localization and coherent backscattering of photons in disordered media. Phys. Rev. Lett. 1985;55:2696-2699.

[7] van Tiggelen BA, Maynard R. Reciprocity and coherent backscattering of light. In: Papanicolaou G, editor. Wave propagation in complex media. New York (NY): Springer; 1998. p. 247-271.

[8] Akkermans E. Mesoscopic physics of electrons and photons. Cambridge: Cambridge University Press; 2007.

[9] Anderson P. Absence of diffusion in certain random lattices. Phys. Rev. 1958;109:1492-1505.

[10] Wiersma DS, Bartolini P, Lagendijk A, Righini R. Localization of light in a disordered medium. Nature. 1997;390:671-673.

[11] $\mathrm{Hu} \mathrm{H}$, Strybulevych A, Page J, Skipetrov S, van Tiggelen B. Localization of ultrasound in a three-dimensional elastic network. Nature Phys. 2008;4:945-948.

[12] Pine D, Weitz D, Chaikin P, Herbolzheimer E. Diffusing wave spectroscopy. Phys. Rev. Lett. 1988;60:1134-1137.

[13] Cowan M, Jones I, Page J, Weitz D. Diffusing acoustic wave spectroscopy. Phys. Rev. E. 2002;65:066605.

[14] Poupinet G, Ellsworth W, Frechet J. Monitoring velocity variations in the crust using earthquake doublets: An application to the calaveras fault, california. J. Geophys. Res. 1984;89:5719-5731.

[15] Snieder R, Grêt A, Douma H, Scales J. Coda wave interferometry for estimating nonlinear behavior in seismic velocity. Science. 2002;295:2253-2255.

[16] Lobkis O, Weaver R. Coda-wave interferometry in finite solids: Recovery of p-to-s conversion rates in an elastodynamic billiard. Phys. Rev. Lett. 2003;90:254302.

[17] Larose E, de Rosny J, Margerin L, Anache D, Gouedard P, Campillo M, van Tiggelen B. Observation of multiple scattering of $\mathrm{khz}$ vibrations in a concrete structure and application to monitoring weak changes. Phys. Rev. E. 2006;73:016609.

[18] Brenguier F, Shapiro N, Campillo M, Ferrazzini V, Duputel Z, Coutant O, Nercessian A. Towards forecasting volcanic eruptions using seismic noise. Nat. Geosci. 2008;1:126-130.

[19] Brenguier F, Campillo M, Hadziioannou C, Shapiro N, Nadeau R, Larose E. Postseismic relaxation along the san andreas fault at parkfield from continuous seismological observations. Science. 2008;321:1478-1481.

[20] Feng S, Sornette D. Acoustical nondestructive evaluation of heterogeneous materials in the multiple scattering regime. J. Acoust. Soc. Am. 1991;90:1742-1748.

[21] Vanneste C, Feng S, Sornette D. Nondestructive evaluations in multiple scattering media. J. Acoust. Soc. Am. 1993;93:2311-2312.

[22] van Rossum M, Nieuwenhuizen T. Multiple scattering of classical waves: microscopy, mesoscopy, and diffusion. Rev. Mod. Phys. 1999;71:313-371.

[23] Nieuwenhuizen T, van Rossum M. Role of a single scatterer in a multiple scattering medium. Phys. Lett. A. 1993;177:102-106.

[24] Pacheco C, Snieder R. Time-lapse travel time change of multiply scattered acoustic waves. J. Acoust. Soc. Am. 2005;118:1300-1310.

[25] Larose E, Planes T, Rossetto V, Margerin L. Locating a small change in a multiple scattering environment. Appl. Phys. Lett. 2010;96:204101.

[26] Rossetto V, Margerin L, Planès T, Larose E. Locating a weak change using diffuse waves: theoretical approach and inversion procedure. J. Appl. Phys. 2011;109:034903.

[27] Froment B. Monitoring slight mechanical changes using seismic background noise [PhD thesis]. University of Grenoble; 2011.

[28] Obermann A, Planès T, Larose E, Campillo M. Imaging preeruptive and coeruptive structural and mechanical changes of a volcano with ambient seismic noise. J. Geophys. Res. 2013;118:1-10.

[29] Taflove A, Hagness S. Computational electrodynamics: The finite-difference time-domain method. Incorporated: The Artech House antenna and propagation library. Artech House; 2005.

[30] Courant R, Friedrichs K, Lewy H. On the partial difference equations of mathematical physics. IBM J. Res. Dev. 1967;11:215-234. 
[31] Sheng P. Introduction to wave scattering, localization and mesoscopic phenomena. Vol. 88. Berlin: Springer; 2006.

[32] Leroy V, Derode A. Temperature-dependent diffusing acoustic wave spectroscopy with resonant scatterers. Phys. Rev. E. 2008;77:036602.

[33] Obermann A, Planès T, Larose E, Sens-Schönfelder C, Campillo M. Depth sensitivity of seismic coda waves to velocity perturbations in an elastic heterogeneous medium. Geophys. J. Int. 2013;194:372-382.

[34] Gardiner CW. Stochastic methods. Berlin: Springer; 2009.

[35] Rossetto V. Local time in diffusive media and applications to imaging. Phys. Rev. E. 2013;88:022103.

[36] Chandrasekhar S. Radiative transfer. New York (NY): Dover publications; 1960.

[37] Lagendijk A, Van Tiggelen B. Resonant multiple scattering of light. Phys. Rep. 1996;270: $143-215$.

[38] Margerin L. Introduction to radiative transfer of seismic waves. Geophys. Monograph Ser. 2005;157:229-252.

[39] Shang T, Gao L. Transportation theory of multiple scattering and its application to seismic coda waves of impulsive source. Sci. Sinica. 1988;31:1503-1514.

[40] Sato H. Energy transportation in one- and two-dimensional scattering media: analytic solutions of the multiple isotropic scattering model. Geophys. J. Int. 1993;112:141-146.

[41] Paasschens J. Solution of the time-dependent boltzmann equation. Phys. Rev. E. 1997;56: $1135-1141$.

[42] Crank J. The mathematics of diffusion. New York (NY): Oxford Science Pub.; 1979.

\section{Appendix 1. Green's function of the wave equation and intensity propagator}

The Green's function of the wave equation in a homogeneous medium is noted $g_{0}(\boldsymbol{s}, \boldsymbol{r}, t)$ for a source in $s$ and a receiver in $\boldsymbol{r}$. Its Fourier transform, which corresponds to the Green's function of the homogeneous Helmholtz equation (HHE), is defined by:

$$
G_{0}(\omega, \boldsymbol{s}, \boldsymbol{r})=\int_{-\infty}^{+\infty} g_{0}(\boldsymbol{s}, \boldsymbol{r}, t) e^{i \omega t} d t
$$

In the multiple scattering regime, the Green's function $G(\omega, \boldsymbol{R})$ of the inhomogeneous Helmholtz equation (IHE) can be expanded in a perturbative series [22], that is diagrammatically represented in Figure A1. Its formulation depends on the position and characteristics of every scatterer in the medium, making it in practice impossible to calculate. However, the average IHE Green's function $\bar{G}(\omega, \boldsymbol{R})$ that corresponds to an average over all the possible realizations of the disorder, can be calculated knowing only a few averaged properties of the medium. In the independent scattering approximation (ISA), its expression contains the following decay:

$$
\bar{G}(\omega, \boldsymbol{R}) \propto e^{-\frac{\|\boldsymbol{R}\|}{2 \ell}}
$$

where $\|\boldsymbol{R}\|$ is the distance between the source and receiver and $\ell$ the scattering mean free path. The average IHE Green's function decreases exponentially with the distance relative to the mean free path. In a non-absorbing medium, this attenuation is only due to scattering processes, converting the coherent wave into multiply scattered waves.

When the disorder is fixed (e.g. in solids), averaging over the disorder is difficult and probing the medium with coherent waves is limited to a depth of a few $\ell$. Beyond, the accessible intensity is mainly composed of incoherent waves and shows a diffuse behavior.

By definition, the intensity is proportional to $\bar{p}(\boldsymbol{s}, \boldsymbol{r}, t)$, the ensemble averaged squared modulus of the wavefield:

$$
\bar{p}(\boldsymbol{s}, \boldsymbol{r}, t)=\overline{|g(\boldsymbol{s}, \boldsymbol{r}, t)|^{2}}=\overline{g(\boldsymbol{s}, \boldsymbol{r}, t) g^{*}(\boldsymbol{s}, \boldsymbol{r}, t)}
$$


Following Sheng [31], we obtain in the frequency domain:

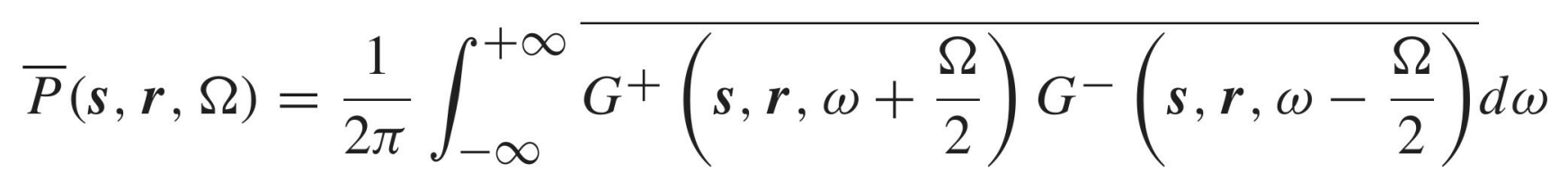

The variable $\omega$ is called internal frequency and describes the fast variations of the wavefield. The variable $\Omega$ (conjugate variable of time $t$ ) is called external frequency and describes the slow variations of the envelope of the signals (or intensity). The + and - indexes denote the retarded (causal) and advanced (acausal) IHE Green's functions.

Considering signals filtered in a narrow frequency band $\Delta \omega$ around $\omega_{0}$ (central angular frequency), we approximate:

$$
\begin{aligned}
\bar{P}(\boldsymbol{s}, \boldsymbol{r}, \Omega) \simeq & \frac{\Delta \omega}{2 \pi} \overline{G^{+}} \overline{\left(\boldsymbol{s}, \boldsymbol{r},-\omega_{0}+\frac{\Omega}{2}\right) G^{-}\left(\boldsymbol{s}, \boldsymbol{r},-\omega_{0}-\frac{\Omega}{2}\right)} \\
& +\frac{\Delta \omega}{2 \pi} G^{+\left(\boldsymbol{s}, \boldsymbol{r}, \omega_{0}+\frac{\Omega}{2}\right) G^{-}\left(\boldsymbol{s}, \boldsymbol{r}, \omega_{0}-\frac{\Omega}{2}\right)} \\
\simeq & \frac{\Delta \omega}{\pi} \overline{G^{+}\left(\boldsymbol{s}, \boldsymbol{r}, \omega_{0}+\frac{\Omega}{2}\right) G^{-}\left(\boldsymbol{s}, \boldsymbol{r}, \omega_{0}-\frac{\Omega}{2}\right)}
\end{aligned}
$$

using the hermitian symmetry of the HHE Green's function $\left(G_{0}^{+}(-\omega)=G_{0}^{-}(\omega)\right)$.

To calculate the product $\overline{G^{+}(\boldsymbol{s}, \boldsymbol{r}) G^{-}(\boldsymbol{s}, \boldsymbol{r})}$, we replace the Green's functions by their perturbative expansions (see Figure A1). In the ISA, the intensity term is written as:

$$
\begin{aligned}
\overline{G^{+}(\boldsymbol{s}, \boldsymbol{r}) G^{-}(\boldsymbol{s}, \boldsymbol{r})}= & \overline{G^{+}}(\boldsymbol{s}, \boldsymbol{r}) \overline{G^{-}}(\boldsymbol{s}, \boldsymbol{r}) \\
& +\iint \overline{G^{+}}\left(\boldsymbol{s}, \boldsymbol{r}_{\mathbf{1}}\right) \overline{G^{-}}\left(\boldsymbol{s}, \boldsymbol{r}_{\mathbf{1}}\right) L_{\Omega}\left(\boldsymbol{r}_{\mathbf{1}}, \boldsymbol{r}_{\mathbf{2}}\right) \overline{G^{+}}\left(\boldsymbol{r}_{\mathbf{2}}, \boldsymbol{r}\right) \overline{G^{-}}\left(\boldsymbol{r}_{\mathbf{2}}, \boldsymbol{r}\right) d \boldsymbol{r}_{\mathbf{1}} d \boldsymbol{r}_{\mathbf{2}}
\end{aligned}
$$

where $L_{\Omega}\left(\boldsymbol{r}_{\mathbf{1}}, \boldsymbol{r}_{\mathbf{2}}\right)$ is called Ladder operator and takes into account all the scattering events between $\boldsymbol{r}_{\mathbf{1}}$ and $\boldsymbol{r}_{\mathbf{2}}$. The external frequency dependence of the Green's functions has been made implicit to alleviate the notations.

This operator satisfies a self-consistent equation known as the Bethe-Salpether equation, represented diagrammatically in Figure A2.

At large times compare to the mean free time (diffusive regime), the spatial variations of the Ladder $L_{\Omega}$ are weak at the mean free path scale $\ell$, contrary to the fast decay of coherent intensity $\overline{G^{+}} \overline{G^{-}}$. We thus use the slowly varying envelope approximation to extract $L_{\Omega}$ from the integral (A6) to write:

$$
G=\text { एका }=\longrightarrow+\longrightarrow
$$

Figure A1. Diagrammatic representation of the perturbative expansion of the IHE Green's function $G$ (hatched line). The thin lines represents the HHE Green's function $G_{0}$ and the dots the potential of the heterogeneities of the medium.

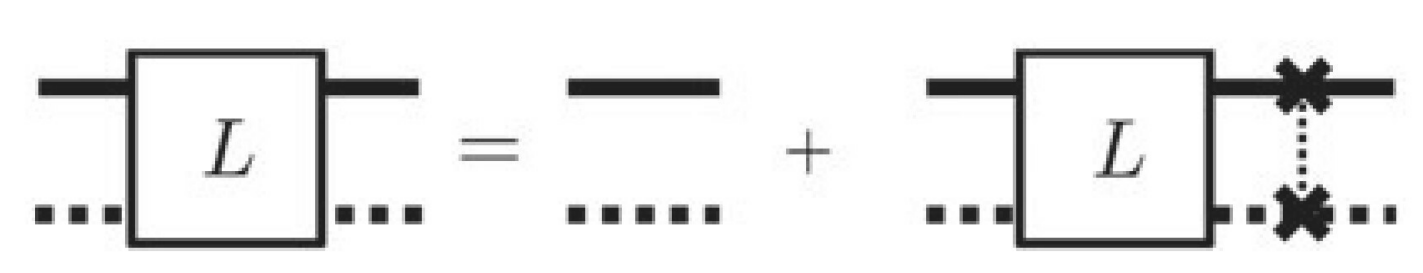

Figure A2. Diagrammatic representation of the Bethe-Salpether equation. The solid (resp. dashed) horizontal lines represent the average retarded (resp. advanced) Green's function $\overline{G^{+}}$(resp. $\overline{G^{-}}$) and the crosses represent the $\boldsymbol{T}$-matrix of the heterogeneities of the medium. The vertical dashed line indicates that the crosses represent the same scatterer. 


$$
\begin{aligned}
\overline{G^{+}(\boldsymbol{s}, \boldsymbol{r}) G^{-}(\boldsymbol{s}, \boldsymbol{r})} & \simeq L_{\Omega}(\boldsymbol{s}, \boldsymbol{r}) \iint \overline{G^{+}}\left(\boldsymbol{s}, \boldsymbol{r}_{\mathbf{1}}\right) \overline{G^{-}}\left(\boldsymbol{s}, \boldsymbol{r}_{\mathbf{1}}\right) \overline{G^{+}}\left(\boldsymbol{r}_{\mathbf{2}}, \boldsymbol{r}\right) \overline{G^{-}}\left(\boldsymbol{r}_{\mathbf{2}}, \boldsymbol{r}\right) d \boldsymbol{r}_{\mathbf{1}} d \boldsymbol{r}_{\mathbf{2}} \\
& =L_{\Omega}(\boldsymbol{s}, \boldsymbol{r}) \int \overline{G^{+}}\left(\boldsymbol{s}, \boldsymbol{r}_{\mathbf{1}}\right) \overline{G^{-}}\left(\boldsymbol{s}, \boldsymbol{r}_{\mathbf{1}}\right) d \boldsymbol{r}_{\mathbf{1}} \int \overline{G^{+}}\left(\boldsymbol{r}_{\mathbf{2}}, \boldsymbol{r}\right) \overline{G^{-}}\left(\boldsymbol{r}_{\mathbf{2}}, \boldsymbol{r}\right) d \boldsymbol{r}_{\mathbf{2}} \\
& =L_{\Omega}(\boldsymbol{s}, \boldsymbol{r})\left(I_{11}\right)^{2}
\end{aligned}
$$

The integral of the coherent intensity is a particular case of loop integrals (Appendix 2). Its value depends on the dimension of the space and is noted $I_{11}$ to remain general. In this regime, it is also known that the Ladder operator follows the diffusion equation [8]:

$$
\left(-i \Omega-D \Delta_{\boldsymbol{r}_{2}}\right) L_{\Omega}\left(\boldsymbol{r}_{1}, \boldsymbol{r}_{2}\right)=\frac{c}{I_{11} \ell} \delta\left(\boldsymbol{r}_{1}-\boldsymbol{r}_{2}\right)
$$

where the diffusion constant is $D=c \ell^{\star} / d, c$ being the energy velocity, $d$ the dimension of space and $\ell^{\star}$ the transport mean free path. In our case of point scatterers in the ISA, we have $\ell=\ell^{\star}$ and we approximate $c=c_{0}$, the phase velocity of the waves. From the diffusion Equation (A8), we define the normalized diffusive intensity $I_{\Omega}^{\text {Diff }}$ :

$$
\begin{aligned}
I_{\Omega}^{\text {Diff }}(\boldsymbol{s}, \boldsymbol{r}) & =\frac{\ell I_{11}}{c} L_{\Omega}(\boldsymbol{s}, \boldsymbol{r}) \\
& =\frac{\ell}{c I_{11}} \overline{G^{+}(\boldsymbol{s}, \boldsymbol{r}) G^{-}(\boldsymbol{s}, \boldsymbol{r})} \\
& =\frac{\ell \pi}{c I_{11} \Delta \omega} \bar{P}(\Omega, \boldsymbol{s}, \boldsymbol{r})
\end{aligned}
$$

In time domain, the diffusive intensity takes the well-known form:

$$
\begin{aligned}
& I_{2 \mathrm{D}}^{\mathrm{Diff}}(R, t)=\frac{1}{(4 \pi D t)} e^{-\frac{R^{2}}{4 D t}} \\
& I_{3 \mathrm{D}}^{\mathrm{Diff}}(R, t)=\frac{1}{(4 \pi D t)^{\frac{3}{2}}} e^{-\frac{R^{2}}{4 D t}}
\end{aligned}
$$

These solutions stand for energy transport in infinite media, at long times compare to the mean free time. They are translationally invariant, depending only on the distance $R=\|\boldsymbol{r}-\boldsymbol{s}\|$.

\section{Appendix 2. Loop integrals}

In Section 3 and Appendix 1, several spatial integrals that involve different combinations of average Green's functions appear. These integrals are easily evaluated in the wavevector domain. Let us first define the spatial Fourier Transform of a function $F(\boldsymbol{R})$ as:

$$
T F_{\boldsymbol{R}}[F(\boldsymbol{R})]=\hat{F}(\boldsymbol{k})=\int F(\boldsymbol{R}) e^{-i \boldsymbol{k} \cdot \boldsymbol{R}} d \boldsymbol{R}
$$

and

$$
F(\boldsymbol{R})=T F_{\boldsymbol{k}}^{-1}[\hat{F}(\boldsymbol{k})]=\frac{1}{(2 \pi)^{d}} \int \hat{F}(\boldsymbol{k}) e^{i \boldsymbol{k} \cdot \boldsymbol{R}} d \boldsymbol{k}
$$

with $d$ the space dimension.

To convert the spatial integrals to the wavevector domain, we also use the following property. Consider $A\left(\boldsymbol{r}, \boldsymbol{r}^{\prime}\right)$ and $B\left(\boldsymbol{r}, \boldsymbol{r}^{\prime}\right)$ two functions depending only on the distance $\boldsymbol{r}-\boldsymbol{r}^{\prime}$ between the two variables. Then the integral:

$$
I=\int A\left(\boldsymbol{r}, \boldsymbol{r}_{\mathbf{1}}\right) B\left(\boldsymbol{r}_{\mathbf{1}}, \boldsymbol{r}^{\prime}\right) d \boldsymbol{r}_{\mathbf{1}}
$$


is rewritten in the wavevector domain $\boldsymbol{k}$ :

$$
\begin{aligned}
I & =\frac{1}{(2 \pi)^{2 d}} \iiint \hat{A}(\boldsymbol{k}) e^{i \boldsymbol{k} \cdot\left(\boldsymbol{r}-\boldsymbol{r}_{1}\right)} \hat{B}\left(\boldsymbol{k}^{\prime}\right) e^{i \boldsymbol{k}^{\prime} .\left(\boldsymbol{r}_{\mathbf{1}}-\boldsymbol{r}^{\prime}\right)} d \boldsymbol{r}_{\mathbf{1}} d \boldsymbol{k} d \boldsymbol{k}^{\prime} \\
& =\frac{1}{(2 \pi)^{2 d}} \iint \hat{A}(\boldsymbol{k}) \hat{B}\left(\boldsymbol{k}^{\prime}\right) \int e^{-i \boldsymbol{r}_{\mathbf{1}} \cdot\left(\boldsymbol{k}-\boldsymbol{k}^{\prime}\right)} d \boldsymbol{r}_{\mathbf{1}} e^{i \boldsymbol{k} \cdot \boldsymbol{r}} e^{-i \boldsymbol{k}^{\prime} \cdot \boldsymbol{r}^{\prime}} d \boldsymbol{k} d \boldsymbol{k}^{\prime} \\
& =\frac{1}{(2 \pi)^{d}} \iint \hat{A}(\boldsymbol{k}) \hat{B}\left(\boldsymbol{k}^{\prime}\right) \delta\left(\boldsymbol{k}-\boldsymbol{k}^{\prime}\right) e^{i \boldsymbol{k} \cdot \boldsymbol{r}} e^{-i \boldsymbol{k}^{\prime} \cdot \boldsymbol{r}^{\prime}} d \boldsymbol{k} d \boldsymbol{k}^{\prime} \\
& =\frac{1}{(2 \pi)^{d}} \int \hat{A}(\boldsymbol{k}) \hat{B}(\boldsymbol{k}) e^{i \boldsymbol{k} \cdot\left(\boldsymbol{r}-\boldsymbol{r}^{\prime}\right)} d \boldsymbol{k}
\end{aligned}
$$

Particularly, for $\boldsymbol{r}=\boldsymbol{r}^{\prime}$, we have:

$$
I=\frac{1}{(2 \pi)^{d}} \int \hat{A}(\boldsymbol{k}) \hat{B}(\boldsymbol{k}) d \boldsymbol{k}
$$

Similarly, we extend the calculation to the integrals:

$$
\begin{aligned}
I & =\int A_{1}\left(\boldsymbol{r}, \boldsymbol{r}_{\mathbf{1}}\right) A_{2}\left(\boldsymbol{r}_{\mathbf{1}}, \boldsymbol{r}_{\mathbf{2}}\right) \ldots A_{n}\left(\boldsymbol{r}_{\mathbf{2}}, \boldsymbol{r}\right) d \boldsymbol{r}_{\mathbf{1}} d \boldsymbol{r}_{\mathbf{2}} \ldots d \boldsymbol{r}_{\boldsymbol{n}-\mathbf{1}} \\
& =\frac{1}{(2 \pi)^{d}} \int A_{1}(\boldsymbol{k}) A_{2}(\boldsymbol{k}) \ldots A_{n}(\boldsymbol{k}) d \boldsymbol{k}
\end{aligned}
$$

When these integrals concern the average Green's functions, they are called loop integrals and we evaluate them following the approach of van Rossum and Nieuwenhuizen [22, Appendix]. Here we use different conventions and we also perform the calculation in 2D. These loop integrals, that appear multiple times in the present study, are defined as:

$$
I_{a b}=\frac{1}{(2 \pi)^{d}} \int \hat{\bar{G}}^{a}(\boldsymbol{k}) \hat{\bar{G}}^{* b}(\boldsymbol{k}) d \boldsymbol{k}
$$

with $d$ the space dimension and where $\hat{\bar{G}}(\boldsymbol{k})=\frac{1}{k_{0}^{2}-n t-k^{2}}$ is the average Green's function of the multiple scattering medium. Defining $\mu^{2}=k_{0}^{2}-n \mathfrak{t}$, we have:

$$
I_{a b}=\frac{1}{(2 \pi)^{d}} \int \frac{1}{\left(\mu^{2}-k^{2}\right)^{a}\left(\mu^{* 2}-k^{2}\right)^{b}} d \boldsymbol{k}
$$

and we obtain the following recurrence relations:

$$
\begin{aligned}
& I_{(a+1) b}=\frac{-1}{2 a \mu} \frac{d}{d \mu} I_{a b} \\
& I_{a(b+1)}=\frac{-1}{2 b \mu^{*}} \frac{d}{d \mu^{*}} I_{a b}
\end{aligned}
$$

If we calculate the simplest integral $I_{11}$, we can deduce the others using the recurrence relations (B8). The integral $I_{11}$ reads:

$$
I_{11}=\frac{1}{(2 \pi)^{d}} \int \frac{1}{\left(\mu^{2}-k^{2}\right)\left(\mu^{* 2}-k^{2}\right)} d \boldsymbol{k}
$$

and thus depends on the space dimension $d$ of the studied medium. 


\section{D case:}

In 3D, the integrand being an even radial function, the integral in spherical coordinates reads:

$$
\begin{aligned}
I_{11} & =\frac{1}{(2 \pi)^{3}} \int_{0}^{+\infty} \frac{k^{2}}{\left(\mu^{2}-k^{2}\right)\left(\mu^{* 2}-k^{2}\right)} d k \int_{0}^{\pi} \sin \theta d \theta \int_{0}^{2 \pi} d \phi \\
& =\frac{1}{(2 \pi)^{2}} \int_{-\infty}^{+\infty} \frac{k^{2}}{\left(\mu^{2}-k^{2}\right)\left(\mu^{* 2}-k^{2}\right)} d k
\end{aligned}
$$

The integrand has four simple poles in $\mu,-\mu, \mu^{*}$, and $-\mu^{*}$. In the ISA, the medium is dilute enough so that we can approximate $\mu$ to the first order on $\frac{1}{k_{0} \ell}$ :

$$
\mu \simeq k_{0}-\frac{n \Re(\mathfrak{t})}{2 k_{0}}+\frac{i}{2 \ell}
$$

where $\mathfrak{t}$ is the $\boldsymbol{T}$-matrix of the scatterers and $\ell$ is the scattering mean free path. We then use the residue theorem integrating in the upper complex plan to obtain:

$$
\begin{aligned}
I_{11} & =\frac{i}{4 \pi\left(\mu-\mu^{*}\right)} \\
& =\frac{\ell}{4 \pi}
\end{aligned}
$$

To calculate $I_{21}$, we use the recurrence relation (B8) to find:

$$
\begin{aligned}
I_{21} & =\frac{-1}{2 \mu} \frac{-i}{4 \pi\left(\mu-\mu^{*}\right)^{2}} \\
& \simeq \frac{-i \ell^{2}}{8 \pi k_{0}}
\end{aligned}
$$

where we assumed that the wavenumber $k_{\text {eff }}=\mu$ of the effective medium does not differ too much from the one of the uniform medium $k_{0}$.

\section{D case:}

In $2 \mathrm{D}$, the integral $I_{11}$ is expressed in polar coordinates:

$$
\begin{aligned}
I_{11} & =\frac{1}{(2 \pi)^{2}} \int_{0}^{+\infty} \frac{k}{\left(\mu^{2}-k^{2}\right)\left(\mu^{* 2}-k^{2}\right)} d k \int_{0}^{2 \pi} d \theta \\
& =\frac{1}{(2 \pi)} \int_{0}^{+\infty} \frac{k}{\left(\mu^{2}-k^{2}\right)\left(\mu^{* 2}-k^{2}\right)} d k
\end{aligned}
$$

Here, the integrand being an odd function, we cannot extend it to the whole real axis. Using the variable substitution $p=k^{2}$, we obtain:

$$
I_{11}=\frac{1}{(4 \pi)} \int_{0}^{+\infty} \frac{1}{\left(p-\mu^{2}\right)\left(p-\mu^{* 2}\right)} d p
$$

and applying a partial fraction decomposition:

$$
\begin{aligned}
I_{11} & =\frac{1}{(4 \pi)} \frac{1}{\mu^{2}-\mu^{* 2}}\left(\int_{0}^{+\infty} \frac{1}{p-\mu^{2}} d p-\int_{0}^{+\infty} \frac{1}{p-\mu^{* 2}} d p\right) \\
& =\frac{1}{(4 \pi)} \frac{1}{\mu^{2}-\mu^{* 2}}\left[\ln \left(p-\mu^{2}\right)-\ln \left(p-\mu^{* 2}\right)\right]_{0}^{+\infty} \\
& =\frac{1}{(4 \pi)} \frac{\ln \left(-\mu^{* 2}\right)-\ln \left(-\mu^{2}\right)}{\mu^{2}-\mu^{* 2}}
\end{aligned}
$$


We define $-\mu^{* 2}=R e^{i \theta}$ and we have $\left|\Im\left(\mu^{2}\right)\right| \ll\left|\Re\left(\mu^{2}\right)\right|$ (dilute medium). We then approximate $\theta \simeq \pi \cdot \operatorname{sgn}\left(k_{0}\right)$ and thus:

$$
\begin{aligned}
I_{11} & =\frac{i \cdot \operatorname{sgn}\left(k_{0}\right)}{2\left(\mu^{2}-\mu^{* 2}\right)} \\
& =\frac{\ell}{4\left|k_{0}\right|}
\end{aligned}
$$

and using (B8):

$$
\begin{aligned}
I_{21} & =\frac{i}{2\left(\mu^{2}-\mu^{* 2}\right)^{2}} \\
& =\frac{-i \ell^{2}}{8 k_{0}\left|k_{0}\right|}
\end{aligned}
$$

